INRA Prod. Anim., 1989, 2 [1], 5-21

\author{
J. THIMONIER, A. SEMPERE \\ INRA Station de Physiologie \\ de la Reproduction \\ Nouzilly 37380 Monnaie \\ * CNRS Centre d'Etudes biologiques \\ des Animaux sauvages \\ Villiers-en-Bois \\ 79360 Beauvoir-sur-Niort
}

\section{La reproduction chez les cervidés}

\section{Résumé}

Les principales caractéristiques de la fonction de reproduction des cervidés ainsi que son contrôle par différents facteurs de l'environnement sont présentés dans cette étude bibliographique.

Sous les latitudes moyennes et élevées, les cervidés originaires de ces latitudes ont un cycle saisonnier très marqué de la reproduction et il y a synchronisme entre l'activité sexuelle des mâles et celle des femelles. Les naissances ont toujours lieu à la fin du printemps ou au début de l'été. Compte-tenu des durées de gestation des différentes espèces présentées ( 200 à 290 jours), les périodes d'accouplement sont très variables d'une espèce à l'autre (été, automne, début de l'hiver), mais fixes pour une espèce donnée.

La photopériode serait l'entraîneur de l'activité sexuelle comme cela a été démontré plus particulièrement chez les cerfs rouges.

Il est possible d'avancer le début de la période de reproduction de 1 à 2 mois (cerfs rouges, daims) par différentes méthodes : effet mâle, traitements progestagène, traitement à la mélatonine. Ces méthodes ne sont toutefois efficaces que dans les 2 mois qui précèdent le début normal de la saison sexuelle.

Sous les latitudes plus faibles et pour les cervidés originaires de ces latitudes, la répartition des mise bas au cours de l'année est beaucoup plus uniforme. Cette caractéristique semble subsister même lorsqu'ils sont transportés sous des latitudes plus élevées.
L'élevage des cervidés pour la production de viande, des peaux, des bois et velours, voire du musc, s'est développé dans différents pays depuis quelques années. Il existe de nombreuses espèces utilisées en élevage ou susceptibles de l'être dans les différentes parties du monde (De Vos 1982). En France, quelques élevages de cerfs rouges, de daims et de chevreuils se sont récemment développés. Au moment où l'INRA met en place un programme de recherches sur l'élevage des cerfs rouges et des chevreuils, une mise au point bibliographique sur la reproduction apparaît nécessaire. Pour une meilleure compréhension de la biologie de la reproduction, cet article ne se limitera pas aux connaissances sur ces seules deux espèces mais sera étendu à celles sur les cervidés vivant soit dans des élevages soit dans des parcs ou réserves zoologiques et sur lesquelles des études ont pu être conduites.

La famille des Cervidae comprend 17 genres, subdivisés en 40 espèces et environ 200 sousespèces (Whitehead 1972). Il ne saurait être question de décrire les caractéristiques de reproduction de chacune d'entre elles. Le tableau 1 indique les espèces qui seront évoquées dans cette étude. 
Tableau 1. Principales espèces évoquées dans l'article.

\begin{tabular}{|c|c|c|c|}
\hline Genre & Nom commun & Nom latin & Nom anglais \\
\hline Cervus & $\begin{array}{l}\text { Cerf rouge } \\
\text { Wapiti } \\
\text { Cerf sika } \\
\text { Cerf de Java } \\
\text { ou Cerf rusa }\end{array}$ & $\begin{array}{l}\text { Cervus elaphus } \\
\text { Cervus canadensis } \\
\text { Cervus nippon } \\
\text { Cervus timorensis }\end{array}$ & $\begin{array}{l}\text { Red deer } \\
\text { Wapiti } \\
\text { Sika deer } \\
\text { Rusa deer }\end{array}$ \\
\hline Axis & Cerf axis & Axis axis & Axis deer \\
\hline Dama & Daim & Dama dama & Fallow deer \\
\hline Rangifer & $\begin{array}{l}\text { Renne } \\
\text { Caribou }\end{array}$ & Rangifer tarandus & $\begin{array}{l}\text { Reindeer } \\
\text { Caribou }\end{array}$ \\
\hline \multirow[t]{2}{*}{ Odocoileus } & $\begin{array}{l}\text { Cerf à queue blanche } \\
\text { ou Cerf de Virginie }\end{array}$ & Odocoileus virginianus & White-tailed deer \\
\hline & $\begin{array}{l}\text { Cerf à queue noire } \\
\text { ou Cerf mulet }\end{array}$ & Odocoileus hemionus & $\begin{array}{l}\text { Black-tailed deer } \\
\text { or Mule deer }\end{array}$ \\
\hline Elaphurus & Cerf du Père David & Elaphurus davidianus & $\begin{array}{l}\text { Père David's deer } \\
\text { or Mi-lu }\end{array}$ \\
\hline Capreolus & Chevreuil & Capreolus capreolus & Roe deer \\
\hline
\end{tabular}

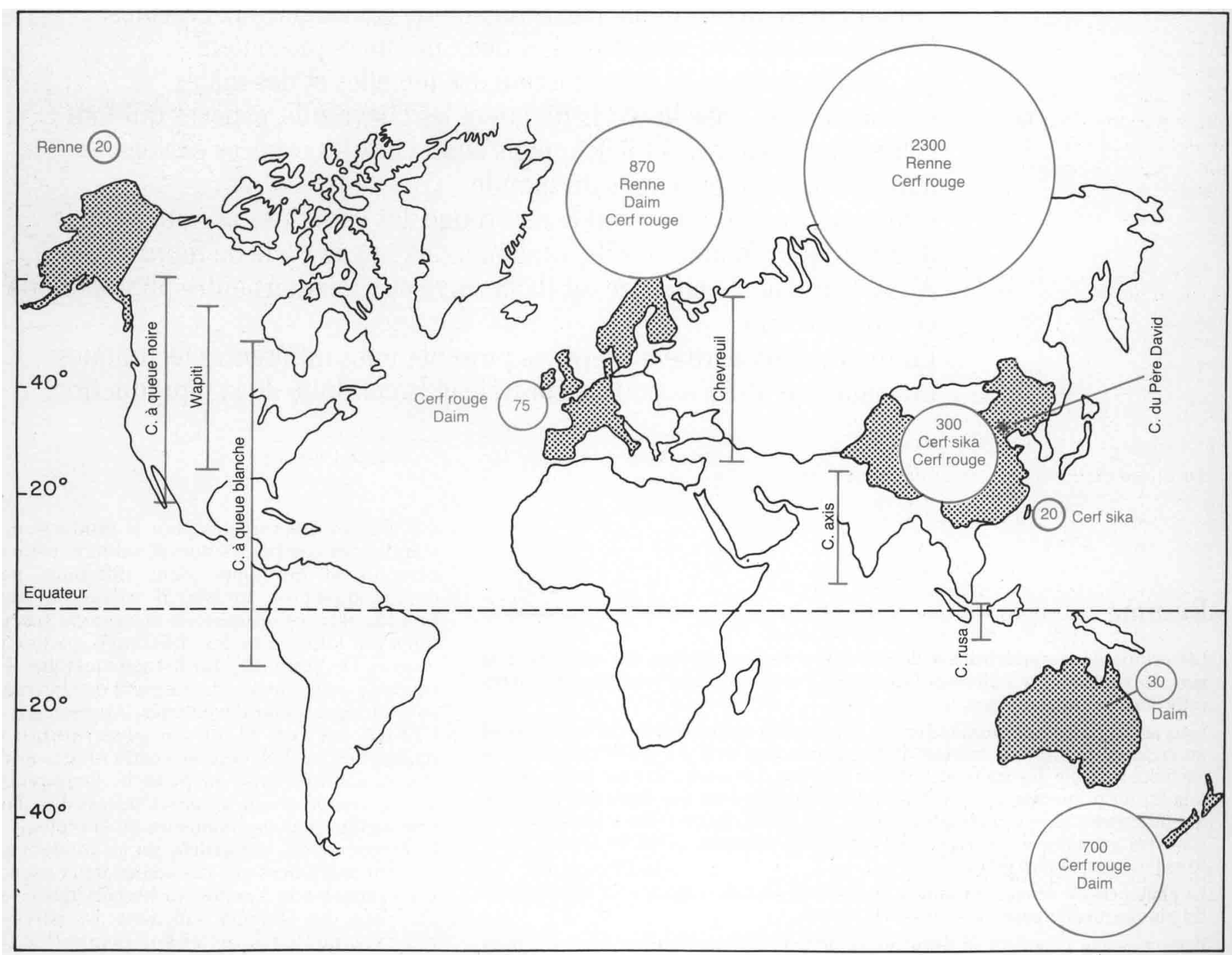

\section{1 / Caractéristiques de reproduction des femelles}

Une des plus récentes revues bibliographiques sur le sujet est celle de Sadleir (1987). Elle comporte plus de 200 références et les caracté- ristiques de reproduction de nombreuses espèces y sont précisées.

\section{1 / Répartition annuelle des mise bas et périodes de reproduction}

a) Dans les régions situées sous des latitudes moyennes et élevées et pour les cervidés origi- 
Tableau 2. Caractéristiques de reproduction de quelques espèces de cervidés sous des latitudes moyennes et élevées.

\begin{tabular}{|l|c|c|c|c|}
\hline \multicolumn{1}{|c|}{ Espèce } & $\begin{array}{c}\text { Période } \\
\text { du rut }\end{array}$ & $\begin{array}{c}\text { Durée gestation } \\
\text { (jours) }\end{array}$ & Prolificité & Référence \\
\hline Cerf de Virginie & Novembre & 200 & 1 à 2 & Plotka et al 1977 a et b \\
Cerf à queue noire & Nov.-Déc. & 203 & 1 à 2 & Thomas et Cowan 1975 \\
Cerf sika & Octobre & 222 & 1 & \\
Daim & Oct.-Nov. & 229 & 1 & \\
Cerf rouge & Octobre & 233 & 1 & De Vos 1982 \\
Renne & Sept.-Oct. & 240 & 1 & \\
Wapiti & Sept.-Oct. & $250-260$ & 1 & Wemmer 1983 \\
Cerf du Père David & Juil.-Août & 288 & 2 & Chapman 1974 \\
Chevreuil & Juil.-Août & $290^{*}$ & & \\
\hline
\end{tabular}

* Espèce à diapause embryonnaire et implantation différée (Short et Hay 1966).

naires de ces régions, les mise bas ont lieu à la fin du printemps (cerfs rouges, daims et chevreuils) ou au tout début de l'été (cerfs rouges et daims) (Fletcher 1974, Kelly et Whateley 1975, Bray et Kelly 1979, Asher et Adam 1985, Lincoln 1985, Kirkwood et al 1987). Près de $90 \%$ des naissances se produisent au cours d'une période dont la durée est inférieure à 1 mois (Kelly et Whateley 1975, Asher et Adam 1985). Le groupement des naissances à la fin du printemps et au début de l'été existe également chez d'autres espèces: rennes, cerfs à queue blanche, cerfs sika, etc.

Les naissances sont très donc tardives par rapport aux disponibilités fourragères qui augmentent bien avant la fin du printemps, tout au moins dans les zones tempérées.

Tout en conservant une période réduite de mise bas, les cerfs du Père David mettent bas beaucoup plus tôt: $75 \%$ des naissances ont lieu au mois d'avril (Kirkwood et al 1987).

La durée de gestation est extrêmement variable d'une espèce à l'autre : de 200 à 290 jours, et les périodes d'accouplement sont très différentes: de juillet-août à novembre-décembre (tableau 2). Il est intéressant de noter que certaines espèces se reproduisent pendant les jours les plus courts (cerfs à queue noire) alors que d'autres (cerfs du Père David, chevreuils) se reproduisent pendant les jours les plus longs, mais, la plupart, quand la durée du jour est décroissante.

Le groupement des mise bas sur une courte durée correspondant à une période des fécondations relativement brève semble conservé quelle que soit la latitude pour les animaux originaires des latitudes moyennes et élevées (cerfs rouges : Fletcher 1974); aussi bien dans l'hémisphère nord que dans l'hémisphère sud, les naissances ont lieu à la même saison (printemps-été). Toutefois, les cerfs de Virginie vivant sous des latitudes très basses peuvent se reproduire quelle que soit la période de l'année (Webb et Nellis 1981, Bronson 1985).

b) Pour les espèces originaires des zones tropicales ou subtropicales et y vivant, des naissances peuvent être observées toute l'année ; il ne semble pas exister de périodes strictes de reproduction. C'est le cas par exemple des cerfs axis et des cerfs de Java.

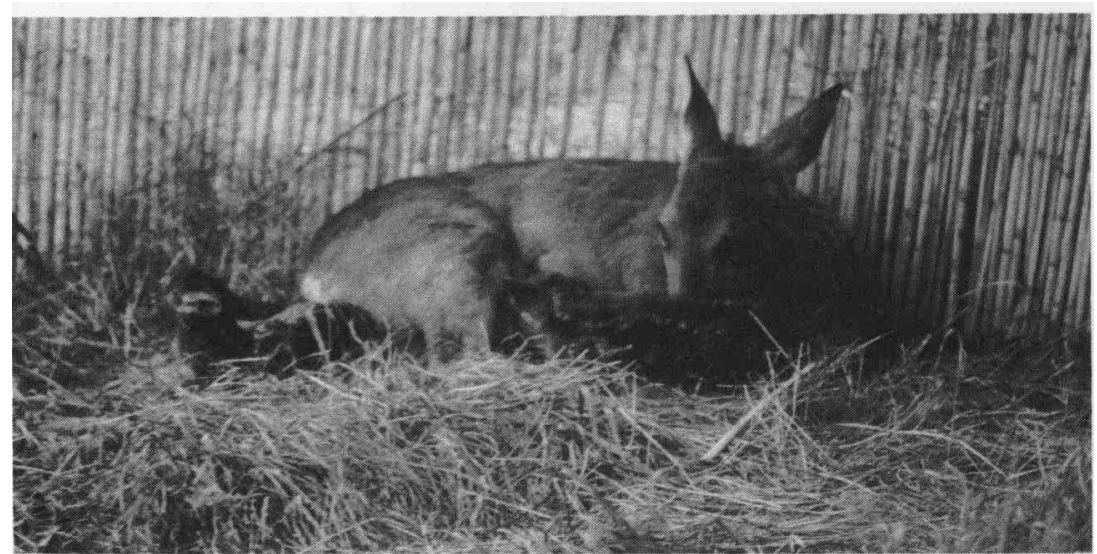

Pour les espèces vivant dans les zones tempérées, les naissances sont groupées à la fin du printemps et au début de l'été.

Cette répartition quasi uniforme des naissances est conservée lorsque ces espèces sont transportées sous des latitudes plus élevées (Lincoln 1985). Il en résulte fréquemment, dans ce cas, une augmentation de la mortalité des jeunes quand les conditions climatiques sont difficiles : elle peut dépasser $50 \%$ (MacKenzie 1985, Kirkwood et al 1987).

Suivant les espèces et en fonction du milieu d'origine, des stratégies différentes ont donc été adoptées par les cervidés pour assurer des mise bas à des périodes favorables à la survie : reproduction en jours courts ou en jours longs, reproduction quelle que soit la période de l'année.

\section{2 / Fertilité et puberté}

\section{a / Femelles adultes}

Chez les cerfs rouges, en Ecosse, dans des conditions naturelles difficiles, la fertilité annuelle des femelles peut varier de 0 à $97 \%$. Elle dépend de l'âge, la puberté n'étant atteinte qu'entre 2 et 4 ans, et de l'état physiologique, les femelles allaitantes étant moins fertiles $(50 \%)$ que les femelles non suitées $(89 \%)$. Un des facteurs les plus importants est le poids, apprécié par le poids de carcasse lors de ces observations : la probabilité d'une femelle adulte de devenir gravide augmente de $10 \%$ 
pour une augmentation de $1,5 \mathrm{~kg}$ du poids de carcasse au-delà d'un poids seuil proche de 40 $\mathrm{kg}$, variant avec l'âge et le fait d'allaiter ou non (Mitchell et Brown 1974).

Dans les élevages, des fertilités proches ou supérieures à $90 \%$ peuvent être obtenues (Kelly et Moore 1977, Hamilton et Blaxter 1980, Asher et Adam 1985, Blaxter et al 1988). Comme dans les conditions naturelles, le facteur le plus important conditionnant la fertilité est le poids lors de la période de reproduction. Il est possible de définir un poids minimum en dessous duquel la fertilité est nulle $(52 \mathrm{~kg})$ et un poids moyen-seuil de $65 \mathrm{~kg}$ pour que la fertilité du troupeau dépasse $50 \%$. Au-delà de 85 $\mathrm{kg}$, plus de $90 \%$ des biches deviennent gravides. Des équations de prédiction de la fertilité en fonction du poids ont ainsi été établies (Hamilton et Blaxter 1980, Blaxter et al 1988) sans que l'âge ne soit pris en compte.

Dans ces conditions, la fertilité est identique que les femelles soient allaitantes ou non. Cependant les femelles allaitantes mettent bas légèrement plus tard (une semaine). Ce retard pourrait être un effet du poids de la mère : chaque diminution de poids de $1 \mathrm{~kg}$ retarde la mise bas de 0,3 jour. Il est possible que la fréquence et la durée des tétées, liées au niveau alimentaire des mères, soient aussi des facteurs retardant l'apparition du premier oestrus (Clutton-Brock et al 1981, Loudon et al 1983).

Chez les daims, la fertilité en élevage peut varier selon les années de 76 à $98 \%$ (Asher et Adam 1985). Comme pour les cerfs, il existe de grandes variations selon les élevages.

Quant aux chevreuils, leur fertilité est mal connue. Toutefois, le fait que plus de $98 \%$ des femelles adultes capturées entre les mois de novembre et mars aient des niveaux élevés de progestérone indiquant une activité lutéale (Sempéré et al 1988), laisse penser que la majorité des femelles ovulent pendant la période de reproduction et peuvent donc devenir gravides. Des phénomènes de surpopulation pourraient non seulement réduire la prolificité dans cette espèce (Chapman 1974) mais également affecter la fertilité.

\section{b / Jeunes femelles : puberté}

La puberté est en général appréciée par l'établissement de la première gestation. Dans les populations sauvages de cerfs rouges pour lesquelles les conditions alimentaires ne sont pas toujours favorables, les pourcentages de femelles mettant bas à l'âge de 2 ans sont relativement faibles et variables d'une année à l'autre (Mitchell 1973, Guinness et al 1978).

En élevage, le poids des jeunes femelles âgées de 16 mois environ lors de la période de reproduction est le seul facteur qui conditionne leur fertilité (Hamilton et Blaxter 1980, Moore et al 1985, Blaxter et al 1988). Les différences de fertilité entre femelles adultes et « pubères » (Bray et Kelly 1979) peuvent s'expliquer par les différences de poids.

En fait, pour être pubères et fertiles, les biches doivent atteindre un poids seuil qui représente environ $75 \%$ de leur poids adulte (Kelly et Moore 1977).
Chez les autres espèces, daims, chevreuils, rennes, les rares données bibliographiques semblent indiquer les mêmes phénomènes et les femelles peuvent être pubères et fertiles à l'âge de 12-14 mois ou 16 mois (daims, rennes) si leur croissance et leur poids sont suffisants. Chez le chevreuil, près de $90 \%$ des femelles âgées de 1 an ont une activité lutéale (Sempéré et al 1988).

Quoi qu'il en soit chez les animaux originaires des zones tempérées, la puberté chez les femelles coïncide toujours avec la saison normale de reproduction des femelles adultes bien que les jeunes femelles mettent bas légèrement plus tard.

Chez les espèces connues pour se reproduire quelle que soit la période de l'année, les données bibliographiques sont pratiquement inexistantes. Il est probable que le poids des animaux, l'état des réserves corporelles et leur évolution jouent aussi un rôle important pour contrôler le moment de la reproduction.

\section{3 / Cycles sexuels et saison de reproduction}

\section{a / Durée du cycle et de la saison}

Pour certaines espèces, la durée des cycles est assez mal connue. La gravidité et l'anoestrus sont, en effet, les états physiologiques habituellement rencontrés et le nombre de cycles est très limité dans les conditions naturelles et en élevage. Depuis quelques années, en particulier en Grande-Bretagne et en NouvelleZélande, des études sur des femelles gardées non gravides se sont développées.

La détection des chaleurs est faite avec des mâles entiers vasectomisés, par observation directe du comportement d'oestrus, ou indirecte avec harnais marqueurs (Guinness et al 1971, Asher 1985, Curlewis et al 1988). L'observation du changement de comportement (activité) des femelles est aussi un excellent critère (Plotka et al 1980, Curlewis et al 1988).

Chez les cerfs rouges, la saison sexuelle peut comporter jusqu'à 8 cycles successifs. La date d'arrêt est beaucoup plus variable (décembre à mars) que celle du début. La durée du cycle oestrien est de $18,3 \pm 1,7$ jours $(m \pm \sigma)$ d'après Guinness et al (1971) et de 18,2 $\pm 2,2$ jours selon Kelly et al (1985). La plupart des cycles ont une durée comprise entre 15 et 23 jours. Des cycles courts de 7 à 9 jours, ou des cycles longs supérieurs à 30 jours, sont quelques fois observés. La durée des cycles augmente du début à la fin de la saison sexuelle et a tendance à diminuer lorsque l'âge des femelles augmente (Guinness et al 1971).

Chez les daims, le nombre d'oestrus au cours de la saison sexuelle varie de 3 à 6 . Les jeunes femelles ont une saison sexuelle plus courte que les adultes (Asher 1985). Tout comme chez les cerfs rouges, la fin de la saison sexuelle semble beaucoup plus variable que le début. La durée du cycle est de $22,4 \pm 1,3$ jours. De même que chez les cerfs rouges, elle augmente au début de la saison sexuelle pour devenir plus variable vers la fin. La durée du cycle ne 
semble pas affectée par l'âge et le poids des femelles.

Chez les cerfs du Père David, la saison sexuelle qui débute pendant les jours les plus longs se termine pendant les jours les plus courts (fin décembre, début février). La durée du cycle est de 19,5 $\pm 2,6$ jours (Curlewis et al 1988).

Plusieurs cycles successifs sont donc possibles chez ces 3 espèces, augmentant ainsi les chances de fécondation. Il en est de même pour de nombreuses autres espèces.

En revanche, il semble certain que les chevreuils ne présentent qu'un seul oestrus (Hoffmann et al 1978, Sempéré et al 1988).

\section{b / Endocrinologie}

L'existence d'une ovulation non associée à un comportement d'oestrus (ovulation silencieuse), d'un cycle court ou d'une activité lutéale avant le premier oestrus de la saison est plus ou moins contestée. Elle pourrait d'ailleurs dépendre de l'espèce. Chez les cerfs rouges, ce phénomène serait pratiquement inexistant (Lincoln et al 1970, Guinness et al 1971, Adam et al 1985) ou concernerait moins de $50 \%$ des femelles (Kelly et Challies 1978) alors que chez les daims, les cerfs du Père David et les cerfs à queue noire, presque toutes les femelles présentent une activité lutéale de courte durée pouvant résulter d'une ovulation silencieuse (Thomas et Cowan 1975, Asher 1985, Curlewis et al 1988). Il en serait de même chez les chevreuils (Hoffmann et al 1978, Schams et al 1980). Plusieurs cycles ovulatoires de courte durée peuvent être observés (daims: Asher 1985).

Au cours de l'oestrus, les concentrations de progestérone sont en général inférieures à 0,5 ou $1 \mathrm{ng} / \mathrm{ml}$ (cerfs de Virginie: Plotka et al 1980 ; cerfs du Père David : Curlewis et al 1988 ; daims : Asher 1985, Asher et al 1986). Chez les cerfs rouges, les données sont rares et contradictoires : les concentrations de progestérone sont faibles pendant la période péri-oestrale (inférieures à $1 \mathrm{ng} / \mathrm{ml}$ ) d'après Adam et al (1985), très variables et pouvant atteindre 4,7 $\mathrm{ng} / \mathrm{ml}$ d'après Kelly et al (1985). Pour ces auteurs, des niveaux variables et élevés lors de l'oestrus pourraient être liés à l'existence de corps jaunes accessoires (Guinness et al 1971, Kelly et Challies 1978) ou à la persistance d'un corps jaune fonctionnel. Ils pourraient avoir également une origine surrénalienne.

Dès le 4" jour après l'oestrus, les niveaux plasmatiques de progestérone sont supérieurs à $1 \mathrm{ng} / \mathrm{ml}$. Les niveaux les plus élevés (3 à 15 $\mathrm{ng} / \mathrm{ml}$ suivant les espèces et les conditions de milieu) sont atteints pendant la deuxième moitié de la phase lutéale. Ils diminuent ensuite rapidement (lutéolyse) en fin de cycle. L'utérus est impliqué dans cette régression: chez le daim, l'hystérectomie le $13^{\mathrm{e}}$ jour du cycle entraîne le maintien des corps jaunes pendant plusieurs mois (Asher et al 1988a).

Toutefois, des phases lutéales longues pouvant correspondre à des corps jaunes persistants (environ 50 jours) ont été observées chez les cerfs du Père David (Curlewis et al 1988).
Chez les chevreuils, les corps jaunes se maintiennent fonctionnels, c'est-à-dire sécrétant de la progestérone, pendant 8 à 9 mois (Hoffmann et al 1978, Schams et al 1980). Il s'agit d'une véritable pseudo-gestation.

Les mécanismes de la régression lutéale peuvent donc être différents d'une espèce de cervidés à l'autre.

Des ovulations silencieuses et des cycles courts peuvent également exister en fin de saison sexuelle (daims, Asher 1985).

Lors d'une superovulation induite, en particulier chez les cerfs rouges (espèce normalement mono-ovulante), les niveaux de progestérone au cours du cycle induit ou au tout début de la gestation sont significativement plus élevés (Kelly et al 1982, Adam et al 1985, Asher et Smith 1987). Les études sur des espèces naturellement polyovulantes sont par trop limitées, voire inexistantes, pour préciser les relations pouvant exister entre nombre d'ovulations et niveaux de progestérone plasmatique. Toutefois, une étude récente a permis de mettre en évidence une corrélation positive entre le nombre d'embryons et la progestéronémie chez le chevreuil (Sempéré et al, résultats non publiés).

Un pic préovulatoire de LH est détecté lors de l'oestrus (cerfs rouges, Kelly et al 1985 ; cerfs de Virginie, Plotka et al 1980 ; daims, Asher et al 1986). Les concentrations d'oestrogènes (oestradiol) augmentent entre la fin de la phase lutéale et l'oestrus (cerfs de Virginie, Plotka et al 1980 ; daims, Asher et al 1986). Chez les cerfs rouges, durant la période péri-oestrale, les concentrations d'oestradiol seraient 4 à 10 fois supérieures $(120 \mathrm{pg} / \mathrm{ml})$ à celles notées dans les autres espèces d'ongulés pour permettre l'oestrus, malgré les niveaux parfois élevés de progestérone (Kelly et al 1985).

\section{4 / Gestation}

a) Chez tous les cervidés, excepté le chevreuil, pendant la majeure partie de la gestation, les concentrations plasmatiques de progestérone restent voisines des concentrations maximales mesurées pendant le cycle, y compris chez les femelles à polyovulation induite (Plotka et al 1977a, Kelly et al 1982, Plotka et al 1982. Adam et al 1985. Asher et Smith 1987). Le ou les corps jaunes semblent être la source principale de progestérone. Ainsi, chez les cerfs de Virginie (Plotka et al 1982), la castration entraîne une diminution de $50 \%$ de la concentration de progestérone périphérique en moins de 30 minutes et des niveaux identiques à ceux de l'anoestrus (inférieurs à $0,5 \mathrm{ng} / \mathrm{ml}$ ) en moins de 3 semaines. Quel que soit d'ailleurs le stade de gestation, la castration entraîne l'avortement (Plotka et al 1982). Cependant, le fait que les niveaux de progestérone soient plus élevés dans la veine utérine que dans l'artère chez certaines femelles gravides suggère que l'utérus et l'unité foeto-placentaire sont capables de sécréter de la progestérone (Plotka et al 1982). Les surrénales pourraient également contribuer à la production de progestérone (Wesson et al 1979).

Chez les cerfs rouges, les niveaux de progestérone commencent à décliner environ 4
Chez le chevreuil, contrairement aux autres cervidés, il n'y a qu'un seul astrus même lorsqu'il n'y a pas fécondation. 
semaines avant la parturition pour atteindre des niveaux de base (moins de $0,5 \mathrm{ng} / \mathrm{ml}$ ) au tout début de la lactation (Kelly et al 1982 , Adam et al 1985).

D’une manière générale, les oestrogènes, oestradiol, oestrone, oestrogènes totaux, augmentent au cours de la gestation et les concentrations les plus élevées sont observées peu de temps avant la parturition (chevreuils, Hoffmann et al 1978 ; cerfs de Virginie, Harder et Woolf 1976, Plotka et al $1977 \mathrm{~b}$; cerfs rouges, Kelly et al 1982). Ils ont probablement une origine foeto-placentaire et leur évolution est tout à fait comparable à celle trouvée chez d'autres ruminants.

Les niveaux de LH pendant la gestation sont comparables à ceux des femelles non gravides. Quant à la prolactine, ses variations semblent plus liées à celles de la photopériode qu'à l'état physiologique : les niveaux sont faibles en hiver et élevés en fin de printemps et en été aussi bien chez les femelles gravides que chez les femelles non gravides (Kelly et al 1982).

b) Le chevreuil est un cas unique chez les cervidés (et les Artiodactyles) : c'est une espèce à ovo-implantation différée. Après fécondation, fin juillet-début août, l'embryon se développe jusqu'au stade blastocyste (environ 1 semaine) puis entre dans une période de dormance, libre dans l'utérus. Ce phénomène est appelé diapause embryonnaire. La reprise du développement et l'implantation dans l'utérus ne s'effectuent qu'après 4 à 5 mois de diapause: il y a implantation différée et gestation proprement dite.

Pendant la diapause, les corps jaunes sécrètent de la progestérone. Au début de la gestation proprement dite, en décembre-janvier, une augmentation significative des niveaux plasmatiques de progestérone, probablement d'origine placentaire, est observée (Sempéré 1977, Hoffmann et al 1978, Aitken 1981, Sempéré 1982, Sempéré et al 1988).

L'élongation des blastocystes après la période de diapause chez les chevreuils est associée à une augmentation significative des niveaux d'oestrogènes (Aitken 1974, Aitken 1981) mais cette augmentation serait plutôt la conséquence que la cause de la croissance embryonnaire rapide. Des injections d'oestradiol induisent une augmentation de la taille des blastocystes mais ne provoquent ni leur différenciation ni leur élongation (Sempéré 1982). Chez les chevreuils non gravides, aucune augmentation des concentrations plasmatiques d'oestrogènes n'est décelable.

\section{5 / Anoestrus}

Dans les conditions naturelles ou d'élevage, la durée réelle de l'anoestrus saisonnier est inconnue chez les femelles non gravides après la période de reproduction. L'absence de gestation peut être liée à une non fécondation ou à l'anoestrus dans les cas d'insuffisance alimentaire.

L'anoestrus de lactation ou post-partum (femelles non allaitantes) est relativement court (environ 4 mois) chez les femelles bien alimen- tées puisqu'elles sont capables d'être fécondées dès la période de reproduction suivant la mise bas. En fait, la durée exacte de l'anoestrus postpartum est inconnue, compte-tenu de l'interaction avec l'anoestrus saisonnier.

L'anoestrus est caractérisé par des niveaux faibles de progestérone plasmatique (inférieurs à $0,5 \mathrm{ng} / \mathrm{ml}$ ) quelle que soit l'espèce (cerfs rouges, Adam et al 1985 ; daims, Asher 1985, Asher et Smith 1987 ; cerfs de Virginie, Plotka et al 1977a ; cerfs du Père David, Curlewis et al 1988). Il en est de même chez les chevreuils lorsque les corps jaunes de "pseudogestation» ont régressé (Sempéré 1977, Hoffmann et al 1978). Quant aux oestrogènes, leurs concentrations restent faibles et varient très peu (cerfs rouges, Kelly et al 1982; cerfs de Virginie, Plotka et al 1977a ; chevreuils, Hoffmann et al 1978).

\section{6 / Caractéristiques ovariennes particulières}

Pendant la gestation, un corps jaune accessoire est trouvé chez près de $50 \%$ des cerfs rouges. Il a une taille inférieure à celle du corps jaune de gestation. Il se forme au début de la gestation et semble se maintenir pendant toute sa durée (Kelly et Challies 1978). De tels corps jaunes ne semblent pas exister dans toutes les espèces (daims par exemple). Ils ont été décrits chez le renne : leur nombre peut être variable, 1 à 3 , et ils sont rencontrés chez près de $70 \%$ des femelles gravides. Alors que le corps jaune de gestation est toujours situé du côté de la corne utérine gravide, les corps jaunes accessoires ont une répartition aléatoire entre les deux ovaires. Si le corps jaune de gestation régresse à la parturition, les corps jaunes accessoires semblent se maintenir pendant la lactation jusqu'à la première ovulation post-partum (Leader-Williams et Rosser 1983). Le rôle de ces corps jaunes accessoires est inconnu.

Chez les cerfs à queue noire, des cycles de croissance folliculaire sont observés bien avant la première ovulation. La lutéinisation de ces follicules peut être observée juste avant le début de la saison de reproduction. Les cycles folliculaires se poursuivent pendant la gestation (Thomas et Cowan 1975).

Mais le cas le plus intéressant est certainement celui des chevreuils chez lesquels les corps jaunes résultant de l'ovulation se maintiennent pendant plusieurs mois, que les femelles aient été saillies ou non (Hoffmann et al 1978, Schams et al 1980).

\section{7 / Parturition}

Les observations ont été faites chez les cerfs rouges. Peu avant la mise bas, les femelles cherchent à s'isoler du troupeau (Clutton-Brock et Guinness 1975). Les symptômes les plus sûrs sont l'apparition des contractions abdominales et utérines qui précèdent la naissance ellemême de 19 à 350 minutes selon Arman et al (1978) et de 2 à 43 minutes d'après Kelly et Whateley (1975). L'apparition des enveloppes foetales précède la mise bas de moins d'une heure. 
La femelle met bas debout ou couchée. La délivrance a lieu en moyenne $1 \mathrm{~h} 30$ après. Les enveloppes sont généralement ingérées par la mère.

Dès la naissance, le jeune est léché par la mère. La station debout est observée entre 12 et 80 minutes après la naissance et la première tétée réussie intervient quelques minutes après (2 à 25 minutes).

Les cas de mortalité des jeunes sont surtout notés dans les quelques jours qui suivent la naissance. Leur nombre varie suivant les conditions de mise bas et l'environnement (11\% des naissances selon Arman et al (1978); $25 \%$ d'après Kelly et Whateley (1975)).

Les conditions de mise bas chez les autres espèces sont assez mal connues.

\section{2 / Caractéristiques de reproduction des mâles}

Le rythme annuel de la croissance et de la chute des bois, structure osseuse caduque, apanage des mâles chez les cervidés à l'exception des rennes et des caribous, (espèces chez lesquelles la femelle porte également des bois) et sa liaison avec la reproduction ont suscité de nombreuses études.

\section{1 / Les bois : un caractère sexuel secondaire}

Chez tous les cervidés, la première apparition des bois est d'abord précédée par la formation des pivots. Ceux-ci, contrairement aux bois, sont persistants et forment un prolongement de la boîte crânienne.

L'endocrinodépendance de la formation de ces pivots a été mise en évidence chez le chevreuil (Sempéré 1982): leur phase de croissance, qui se situe dès les premiers mois après la naissance, est concomitante d'un accroissement significatif de la testostérone. Si la castration inhibe le développement des pivots, l'injection de testostérone chez un jeune castrat ne rétablit pas cependant leur développement (Blauel 1936).

Chez le chevreuil, cette formation des pivots est souvent suivie d'une pousse de petits bois (broches) qui tombent dès l'hiver et de nouveaux bois apparaissent ensuite (Sempéré 1982 : figure 1). Ainsi le jeune chevreuil peut avoir 2 cycles de bois au cours de la première année de vie. Toutefois, certains chevreuils de poids réduit ne présentent pas la formation de telles broches bien que les pivots existent.

L'existence de 2 cycles des bois au cours de la première année de vie est également observée chez les rennes et les cerfs de Virginie (Leader-Williams 1979, Brown et al 1983). En revanche, les cerfs rouges, les daims ne présentent leur "première tête » qu'à l'âge de 18 mois.

Sous les latitudes moyennes et élevées, chez les cerfs rouges, la chute des bois a lieu au tout début du printemps. Elle est immédiatement
Figure 1. Evolution pondérale et cycle des bois au cours de la première annee de vie chez le chevreuil (Sempéré 1982).

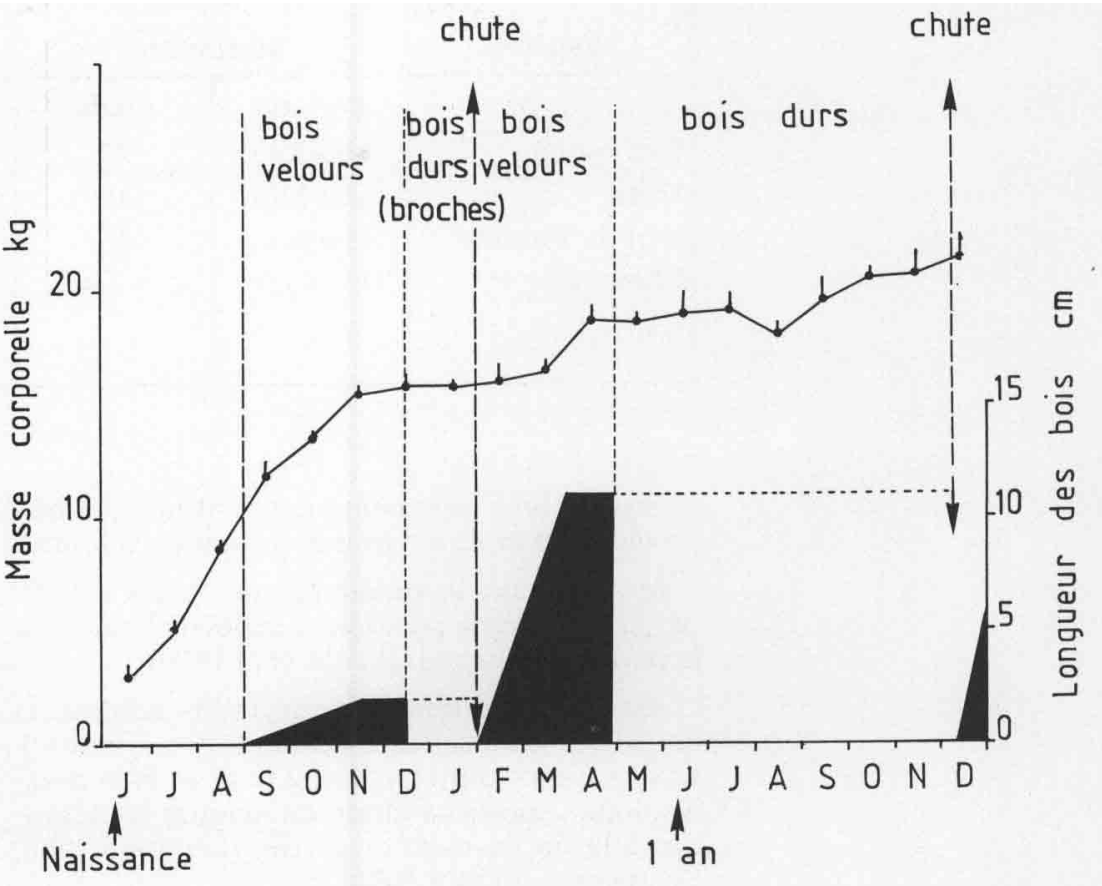

suivie du développement de nouveaux bois à partir soit du pivot, soit du tissu osseux ou de la peau adjacents au pivot (Goss 1985). Pendant toute leur croissance les bois sont irrigués et protégés par le velours qui se dessèche et tombe en lambeaux à la fin de l'été. Cependant, les premiers bois apparaissant à l'âge de 1 an restent en velours jusqu'au mois de novembre. Ils chutent aussi plus tardivement que chez les animaux adultes. La dimension des bois augmente d'année en année jusque vers l'âge de 8 ans.

Les changements anuluels sont sous le contrôle des sécrétions testiculaires. Chez les animaux adultes, les conséquences de la castration dépendent du stade de développement des bois. En velours, ils subsistent pour le reste de la vie du cerf. Minéralisés, ils chutent, mais de

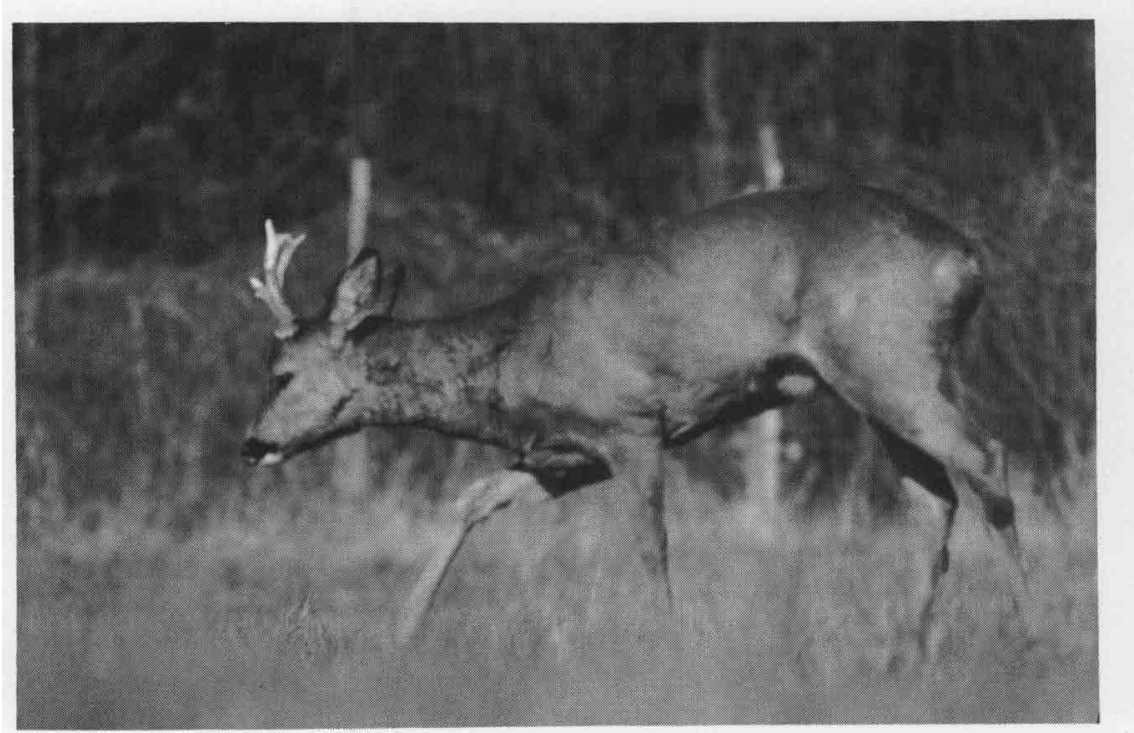


Tableau 3. Caractéristiques testiculaires chez quelques cervidés des latitudes moyennes et élevées.

\begin{tabular}{|l|c|c|c|c|l|}
\hline \multirow{1}{*}{ Espèce } & \multicolumn{4}{|c|}{ Poids d'un testicule (g) } & \multicolumn{1}{c|}{ Référence } \\
\hline \multirow{3}{*}{ Cerf rouge } & \multicolumn{2}{|c|}{ Minimum } & \multicolumn{2}{c|}{ Maximum } & \\
Daim & Période & Poids & Période & Poids & \\
Cerf de Virginie & Mai-Juin & 20 & Sept. & 85 & Lincoln 1971 \\
Chevreuil & Mai-Juin & 10 & Oct.-Nov. & 65 & Chaplin et White 1972 \\
& Mai-Juin & 10 & Oct.-Nov. & 45 & Mirarchi et al 1977 \\
& Déc.-Janv. & 3 à 4 & Juil.-Août & 25 & $\left\{\begin{array}{l}\text { Short et Mann 1966 } \\
\text { Bramley 1970 } \\
\text { Sempéré 1982 }\end{array}\right.$ \\
& & & & & \\
\hline
\end{tabular}

nouveaux bois repoussent pendant une période proche de 4 mois environ et restent en velours.

Inversement, le traitement de mâles castrés par la testostérone provoque le dessèchement et la perte du velours (Lincoln et al 1970).

Au contraire, chez les chevreuils adultes, la chute des bois a lieu en automne (novembre). La croissance dure environ 2 mois et la minéralisation, 1 mois. La chute du velours est terminée à la fin du mois de février (Sempéré 1982, Sempéré et Boissin 1982).

Ces 2 exemples, cerf rouge et chevreuil, illustrent bien les variations qui existent d'une espèce à une autre, mais pour une espèce donnée, le cycle des bois est synchronisé chez l'ensemble des individus.

Au contraire, s'il subsiste bien un cycle circannuel des bois chez les animaux originaires des faibles latitudes (et y vivant), il n'existe aucune synchronisation entre les individus (Goss et Rosen 1973). Chez ces espèces, la période de la chute des bois et la formation de nouveaux trophées dépend surtout de la date de naissance et reste constante par la suite. Transportés sous des latitudes moyennes ou élevées, cet asynchronisme est conservé (Loudon et Curlewis 1988).

De nombreux autres caractères sexuels secondaires (glandes, pelage, diamètre du cou,..) varient aussi au cours du cycle annuel de reproduction.

\section{2 / Activité testiculaire et sécrétions hormonales}

\section{a / Activité testiculaire}

Sous les latitudes moyennes et élevées, et pour les animaux originaires de ces latitudes, le volume et le poids des testicules subissent des variations saisonnières importantes (tableau 3 ). Le poids testiculaire est maximal alors que les bois ont perdu leur velours. Ce maximum coincide avec le début de la saison de reproduction des femelles. Il est minimal peu de temps après la chute des bois.

Dans toutes les espèces, la croissance testiculaire, appréciée par le poids ou le volume, le diamètre antéro- postérieur ou la circonférence scrotale, est décelable entre 3 et 5 mois avant la période de rut. La régression testiculaire débute soit pendant, soit juste après le rut : cerf rouge, Lincoln 1971, Barrell et al 1985 ; wapiti, Haigh et al 1984; chevreuil, Short et Mann 1966 , Bramley 1970, Sempéré 1982 ; daim, Chaplin et White 1972 ; cerf à queue blanche, McMillin et al 1974, Mirarchi et al 1977 ; renne, Leader-Williams 1979.

Ces changements testiculaires résultent des variations de l'activité spermatogénétique. Ainsi, chez le chevreuil, aucune production de cellules germinales différenciées (spermatocytes et spermatides] n'est décelable en janvier. En revanche, dès la fin du mois de mai, l'activité spermatogénétique est élevée et des spermatozoïdes sont trouvés dans la lumière des tubes séminifères. Les premières dégénérescences apparaissent au mois de septembre. L'arrêt de la production de sperme, révélé par l'absence de spermatozoïdes dans la tête de l'épididyme, a lieu au mois d'octobre. Les cellules de Leydig (sécrétion de testostérone) suivent également un cycle annuel. Des signes d'involution de ces cellules sont décelables dès le mois d'août (Short et Mann 1966).

Chez les certs rouges, les rendements des divisions spermatogoniales, de la méïose et de la spermiogénèse sont plus faibles au printemps qu'à l'automne (Hochereau de Reviers et Lincoln 1978). D'après ces auteurs, la longueur des tubes séminifères varie du simple $(1,2 \mathrm{~km}$ au printemps) au double $(2,4 \mathrm{~km}$ en automne $)$ De telles variations dans l'efficacité de la spermatogénèse se traduisent par l'absence parfois complète de spermatozoïdes dans l'épididyme ou dans l'éjaculat (cerf à queue blanche : Mirarchi et al 1977, wapiti : Haigh et al 1984, etc).

Dans les différentes espèces, les mâles seraient d'ailleurs infertiles lorsque le poids des testicules représente moins de $25 \%$ de celui observé au début de la période de rut (Lincoln 1985).

Chez les cerfs axis, originaires des latitudes basses mais élevés sous des latitudes movennes, le volume testiculaire peut varier de 1 à 5 (de $17,8 \pm 1,8$ à $87,2 \pm 6,6 \mathrm{~cm}^{3}$ ) au cours de l'année. Cependant, comme pour les bois, il n'y a pas de synchronisation entre les individus. Néanmoins, le volume testiculaire minimal est observé peu de temps après la chute des bois et le maximum, 2 à 3 mois après la perte du velours. L'azoospermie n'est jamais totale (Loudon et Curlewis 1988). 
Figure 2. Evolutions des taux plasmatiques de $\mathrm{LH}$ et de testostérone chez le jeune chevreuil (Sempéré et Lacroix 1982).

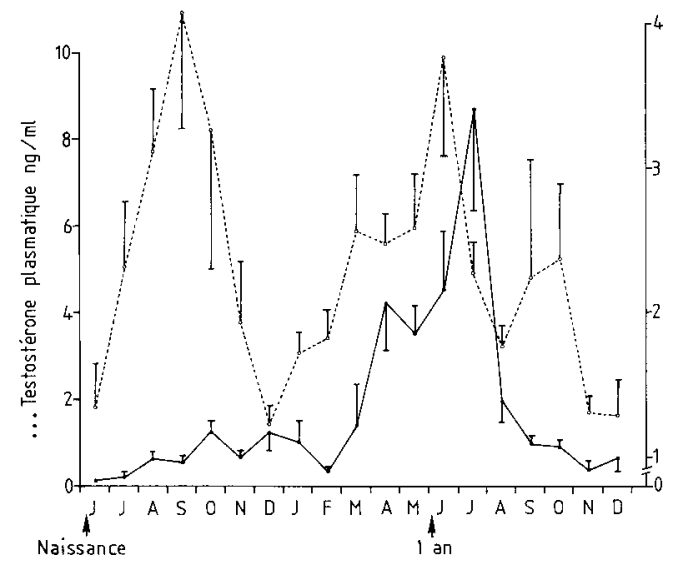

\section{b / Variations hormonales}

Les variations de la fonction exocrine testiculaire sont sous la dépendance des hormones hypophysaires FSH et LH, elles- mêmes contrôlées par le système hypothalamique et le système nerveux central. Il en est de même de la fonction endocrine.

\section{Activité hypophysogonadique}

Avant la puberté, il existe 2 périodes d'activation de la fonction de reproduction pendant la première année de vie chez le chevreuil (Sempéré et Lacroix 1982 : figure 2) et le cerf de Virginie (Brown et al 1983).

Chez le chevreuil, les naissances ont lieu à la fin du printemps. Les concentrations plasmatiques de LH sont importantes dès le mois de septembre. Les valeurs mesurées à cette époque sont équivalentes à celles observées chez l'animal adulte au cours de la période d'activité gonadotrope maximale.

Par ailleurs, les taux plasmatiques de FSH sont élevés durant les mois qui suivent la naissance et le volume testiculaire augmente régulièrement jusqu'en décembre (figure 3). Quand le volume testiculaire atteint ce premier maximum, l'évolution spermatogénétique est bien avancée puisque des images de spermatides sont observées bien que le nombre de spermatozoïdes présents soit toujours faible (Sempéré et al 1983). Les taux plasmatiques de testostérone sont également élevés mais ils sont toujours très inférieurs à ceux observés chez le chevreuil adulte au cours de la période de reproduction (Sempéré et Lacroix 1982 : figure 2 ). Cette première phase d'activité hypophysogonadique est suivie d'une période de repos (fin décembre) avec effondrement des taux plasmatiques de LH, FSH et testostérone et régression du volume testiculaire.

La deuxième activation de la fonction génitale débute 8 mois après la naissance par une augmentation de la concentration plasmatique de LH. Celle-ci est observée en janvier, en même temps que se manifeste chez l'animal adulte la reprise saisonnière du cycle annuel de la fonction gonadotrope.

L'examen des situations temporelles des deux périodes d'activité hypophysogonadique
Figure 3.Evolutions du taux plasmatique de FSH et du volume testiculaire chez le jeune chevreuil (Sempéré 1982).

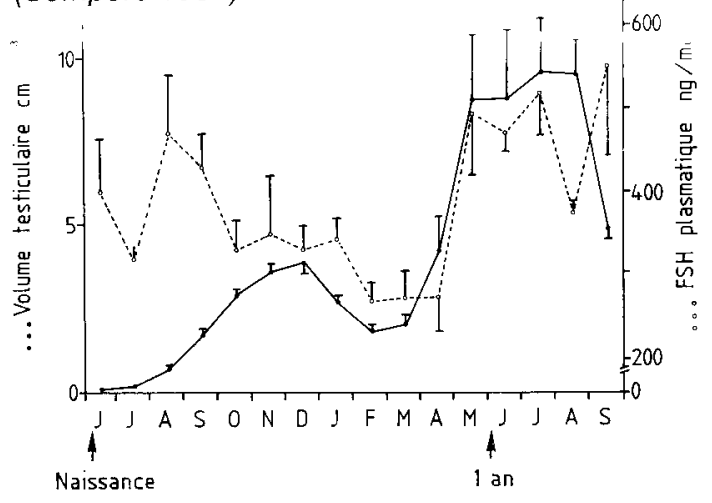

Figure 4. Evolutions des taux plasmatiques de LH et de testostérone chez le chevreuil (Sempéré 1982) et chez le cerf de Virginie (Bubenik et al 1982, Sempéré 1988).

\section{chevreuil}

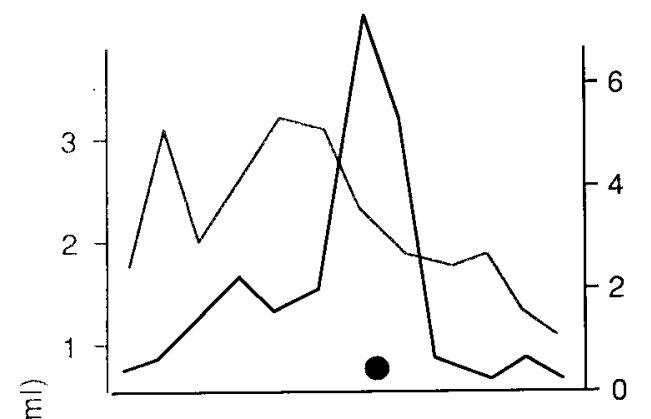

$\frac{\widehat{\bar{E}}}{\text { ঢ }}$
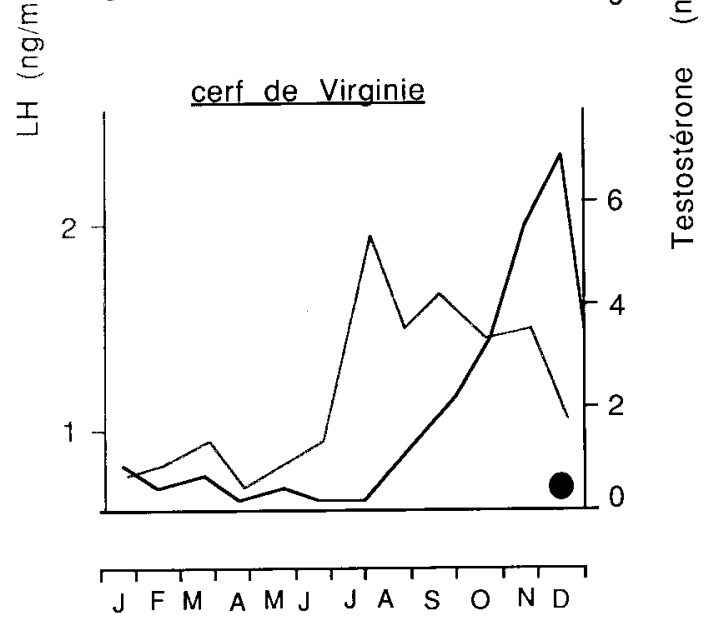

période du rut

au cours de la première année de vie chez le chevreuil ou le cerf de Virginie, indique que la première est concomitante de la diminution de la durée d'éclairement journalier tandis que la seconde, comme celle observée chez le chevreuil adulte, est synchrone de l'augmentation progressive de la durée de la phase claire.

Chez l'adulte, les études portant sur les variations saisonnières des hormones hypophysogonadiques (LH, FSH, testostérone) ont été abordées chez plusieurs espèces de cervidés. 
Chez celles présentant une activité testiculaire maximale en automne (cerf rouge, cerf de Virginie) les sécrétions de LH et FSH sont importantes dès le début de l'été : Bubenik et al 1982 (figure 4).

Les sécrétions de $\mathrm{LH}$ et de testostérone sont pulsatiles et chaque décharge de LH induit une décharge de testostérone (cerf rouge, Lincoln et Kay 1979). Les relations LH - testostérone varient au cours du cycle annuel. Au printemps, pendant la croissance des testicules et des bois, à des décharges pulsatiles de LH de grande amplitude correspondent des décharges de testostérone de faible amplitude. En été, au contraire, à des décharges de LH de faible amplitude correspondent des décharges de testostérone très importantes. Un tel phénomène a été décrit chez le bélier (Pelletier 1986). Les réponses à des injections de GnRH, facteur de décharge des hormones hypophysaires, à ces mêmes périodes sont de mêmes types (Suttie et al 1984a, Fennessy et al 1988).

Chez le chevreuil, qui présente une reprise de l'activité testiculaire entre janvier et juin (photopériode croissante), les relations entre $\mathrm{LH}$ et FSH d'une part et volume testiculaire et testostérone d'autre part, sont du même type que celles observées chez le cerf de Virginie (Sempéré 1988) mais avec un décalage de 6 mois (figure 4). Toutefois, d'importantes variations annuelles du niveau de base de LH et FSH sont enregistrées y compris en automne (septembre-octobre). Des résultats récents obtenus chez cette espèce mettent en évidence, suite à une injection de GnRH, une réponse de L.H très importante en janvier alors qu'elle est négligeable en novembre-décembre (Sempéré et al, résultats non publiés).

\section{Prolactine}

Quelle que soit l'espèce, les variations saisonnières de la prolactinémie présentent des relations directes de phase avec le cycle annuel de la durée d'éclairement journalier ou de la température: maximum en été, minimum en hiver. La prolactine pourrait être impliquée dans le développement des bois. Sa suppression par l'ergobromocryptine (CB 154), traitement toutefois peu spécifique, entraîne une précocité de la minéralisation des bois, de l'augmentation des niveaux de testostérone et de la période de rut chez les cerfs à queue blanche (Bubenik et al 1985, Bubenik 1986). Il reste à savoir s'il s'agit d'une action directe ou indirecte de la prolactine ou du traitement luimême.

Chez le chevreuil, le cycle annuel de la prolactinémie est synchrone des variations saisonnières du volume testiculaire et de la testostéronémie (Schams et Barth 1982, Sempéré et Bois$\sin 1982$ ). La suppression de la prolactine par le CB154 LA (forme retard) se traduit par une régression estivale du volume testiculaire.

\section{3 / Photopériode et activité de reproduction}

Les cervidés, sous les latitudes moyennes et élevées, se reproduisent en général en automne ou en hiver ce qui se traduit par des naissances au moment favorable du point de vue climatique. Néanmoins, parmi les cervidés, le chevreuil occupe une place particulière dans la mesure où le mâle présente un cycle annuel du fonctionnement testiculaire dont la reprise est située entre les mois de janvier et février tandis que la femelle présente des ovulations pendant les jours les plus longs (Sempéré 1982). La figure 5 résume, pour le chevreuil et le cerf rouge, les principales caractéristiques du cycle annuel de reproduction chez les mâles et les femelles. Les cerfs du Père David ont, comme les chevreuils, un cycle annuel de reproduction décalé par rapport à celui des autres cervidés, tout au moins en ce qui concerne les femelles (Curlewis et al 1988). Ainsi, ces 2 espèces, chevreuil et cerf du Père David, présenteraient, visà-vis de la photopériode, une sensibilité différente de celle des autres cervidés.

La photopériode et ses variations sont l'entraîneur principal de l'activité de reproduction chez les petits ruminants originaires des latitudes moyennes et élevées (Thimonier et al 1986). L'information photopériodique est traduite en un signal hormonal, sécrétion de mélatonine, par la glande pinéale. La mélatonine est sécrétée pendant la nuit et c'est par la durée de la sécrétion nocturne de mélatonine que les animaux peuvent mesurer le temps (Karsch et al 1984, Arendt 1986, Collin et al 1988). Il en serait de même chez les cervidés (Bubenik 1986). Chez les ovins, il a été suggéré que les moments de sécrétion de la mélatonine seraient plus importants que la durée de sécrétion pour la lecture de la durée du jour (Ravault et Thimonier 1988). Cette hypothèse n'a pas été vérifiée chez les cervidés.

Plusieurs expériences démontrent le rôle de la photopériode et l'implication de la mélatonine.

Ainsi chez les cerfs rouges mâles, un rythme photopériodique reproduisant en 6 mois les variations annuelles de la photopériode, entraîne 2 périodes de croissance et de régression testiculaires dans l'année. Les testicules ont le volume le plus élevé pendant les jours les plus courts et les plus faibles pendant les jours les plus longs (Pollock 1975, Brown et al 1979, Simpson et al 1983 et 1984). De tels régimes photopériodiques modifient également l'ingestion volontaire, le cycle des bois (Goss 1969) et du pelage (Kay et Ryder 1978) et l'environnement hormonal. Les variations de la prolactinémie suivent celles de la durée de la photopériode claire et celles de la testostérone sont semblables à celles des volumes testiculaires (Brown et al 1979).

L'alternance de périodes de jours longs et de jours courts constants tous les 2 mois entraîne la même alternance de périodes de régression et de croissance testiculaires (croissance pendant les jours courts, régression pendant les jours longs) et affecte également l'ingestion volontaire et les cycles des bois (Suttie et al 1984b)

La pinéalectomie réalisée chez les cerfs de Virginie mâles (Brown et al 1978, Schulte et al 1981, Snyder et al 1983) désynchronise le cycle 
annuel de reproduction, de la prolactinémie et des bois, tout au moins la première année. L'ablation des ganglions cervicaux supérieurs du système sympathique a les mêmes conséquences (Lincoln 1985), l'information photopériodique passant par les ganglions cervicaux supérieurs (Lincoln et Short 1980, Karsch et al 1984). Des facteurs autres que la photopériode (température, alimentation) ou un rythme endogène circannuel pourraient chez les animaux pinéalectomisés ou ganglionectomisés expliquer la synchronisation qui réapparaît audelà de la première année.

L'administration de mélatonine chez les cerfs rouges et les cerfs de Virginie (Bubenik 1983, Lincoln et al 1984, Bubenik et Smith 1985, Bubenik 1986) pendant les jours longs, entraîne l'apparition précoce du rut, de la minéralisation des bois et de la mue ainsi que des changements hormonaux importants: diminution de la prolactinémie, augmentation de LH et testostérone plasmatiques. Cette action de la mélatonine sur l'activité testiculaire passe par une modification de la sécrétion de GnRH : l'immunisation active contre GnRH supprime la réponse testiculaire à l'administration de mélatonine mais n'empêche pas la pousse des bois (Lincoln et al 1984).

Chez les jeunes femelles prépubères, l'application de jours courts après les jours longs de l'été entraîne une apparition plus précoce de la puberté que chez les femelles soumises aux variations normales de la photopériode et ce, aussi bien chez les cerfs rouges (Webster et Barrell 1985) que chez les cerfs à queue blanche (Budde 1983). Ces traitements photopériodiques (application de jours courts) entraînent une diminution de la prolactinémie et induisent la mue du pelage d'été.

L'administration de mélatonine pendant les jours longs de l'été a les mêmes conséquences sur le début de la saison de reproduction, la mue du pelage et la prolactinémie que des jours courts chez les cerfs rouges (Nowak et al 1985, Webster et Barrell 1985).

Le rôle de la photopériode sur le cycle du pelage a été également mis en évidence chez les chevreuils. Son influence sur l'activité de reproduction et en particulier sur la durée de la diapause est cependant non démontrée (Lincoln et Guinness 1972).

En revanche, le fait que, même sous des latitudes élevées, les animaux originaires des faibles latitudes soient capables de se reproduire quelle que soit la période de l'année, pose la question du rôle de la photopériode sur l'activité de reproduction chez ces espèces. D'ailleurs, la croissance testiculaire et la longueur des bois ne semblent pas être modifiées par un traitement « mélatonine » à la fin du printemps chez les cerfs axis en Grande-Bretagne (Loudon et Curlewis 1988).

\section{4 / Contrôle de la reproduction}

Un des objectifs prioritaires en élevage, tout au moins sous les latitudes moyennes et élevées avec des conditions climatiques parfois
Figure 5. Cycles annuels de la reproduction, des caractères sexuels secondaires (bois) et des mues du pelage chez le chevreuil et chez le cerf rouge.
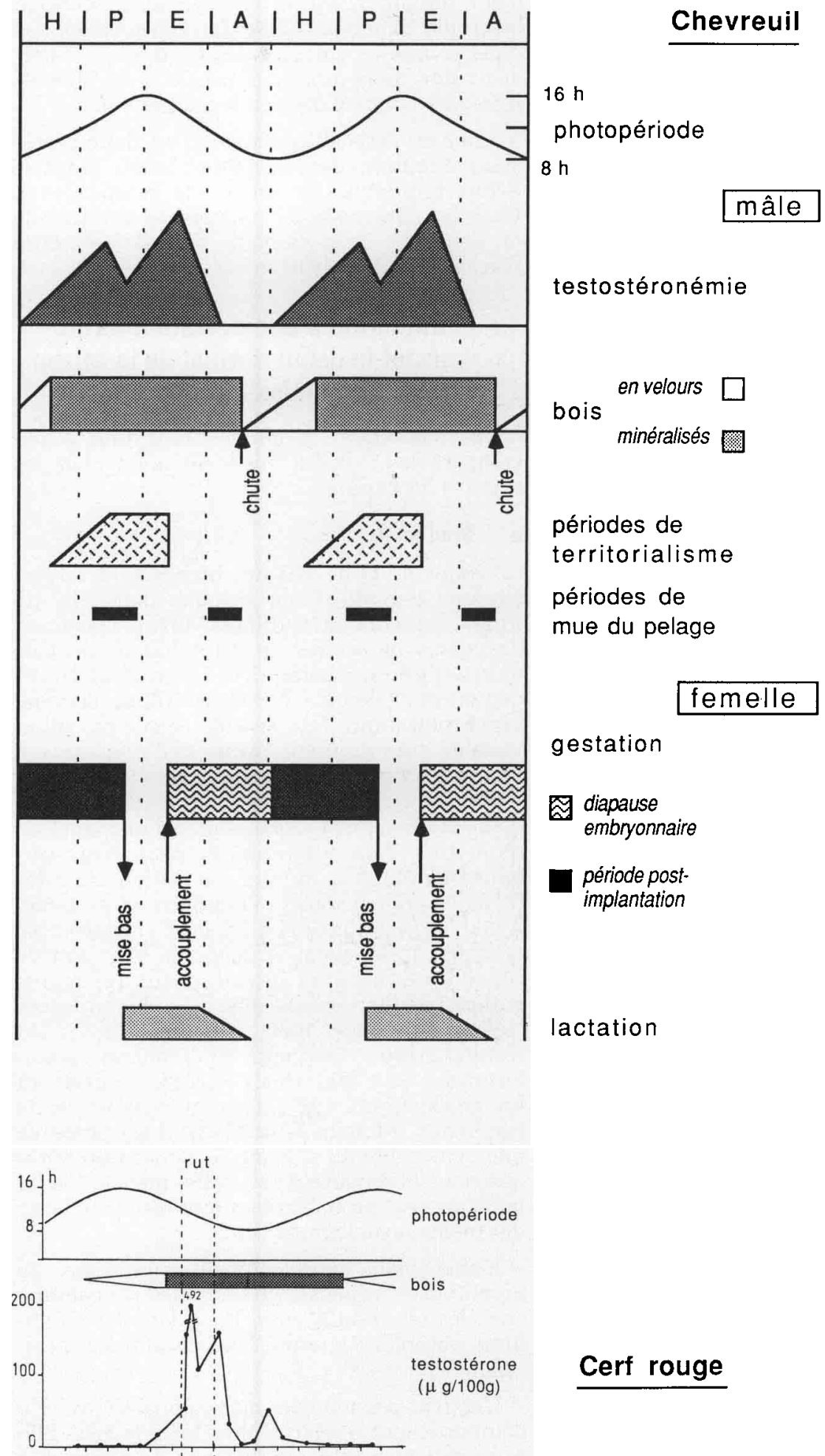

Cerf rouge

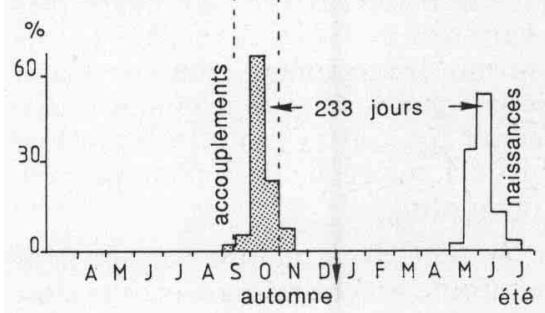


difficiles pendant la période hivernale, est d'avancer la date de mise bas de 1 à 2 mois chez les cerfs, les daims et les chevreuils pour que les jeunes puissent bénéficier de fourrages en quantité et qualité sur une période suffisamment longue avant le sevrage et la période hivernale (Thériez 1988). En outre, dans les espèces mono-ovulantes (cerfs, daims), l'augmentation de la prolificité peut être un objectif si les conditions d'élevage le permettent.

Chez les cerfs et les daims, il est donc nécessaire d'induire une ovulation fertile avant le début normal de la saison de reproduction. Chez les chevreuils, le contrôle de la durée de la diapause embryonnaire devrait permettre d'avancer la date de mise bas.

\section{1 / Induction d'une ovulation fertile avant le début normal de la saison de reproduction}

Les techniques proposées sont tout à fait comparables à celles mises au point chez les ovins et les caprins.

\section{a / Effet mâle}

Cinquante pour 100 des biches sont saillies sur une période d'une semaine dans l'Ile de Rhum (Lincoln et Guinness 1973). L'examen des dates moyennes de mise bas et de leur écart-type en élevage (Blaxter et al 1988) démontre également l'existence d'une certaine synchronisation des oestrus, synchronisation variable d'un groupe à l'autre et d'une année à l'autre. Cette synchronisation serait liée à un " effet mâle », phénomène bien étudié chez les ovins et les caprins chez lesquels l'introduction d'un mâle dans un troupeau peut, dans certaines conditions, induire l'ovulation chez les femelles anovulatoires (Thimonier et al 1986). Ainsi, l'introduction d'un cerf vasectomisé quelques jours avant le début de la saison de reproduction puis l'introduction des mâles entiers résultent en des mise bas plus précoces (Moore et Cowie 1986, McComb 1987). Un résultat presque identique est obtenu en faisant entendre aux femelles l'enregistrement du brame (McComb 1987). Comme chez les petits ruminants (Morgan et al 1972), il est probable que l'ensemble du système sensoriel soit impliqué dans la réponse à cet " effet mâle ». L'effet mâle ne peut probablement être réalisé qu'avec des mâles sexuellement actifs.

L'effet mâle existe probablement dans de nombreuses espèces de cervidés y compris chez les chevreuils, aussi bien dans les conditions naturelles que dans les conditions d'élevage.

L'activité sexuelle des mâles apparaît avant le comportement d'oestrus chez les femelles. Elle pourrait servir d'entraîneur de celle des femelles (Lincoln et Guinness 1973). Les femelles peuvent inversement stimuler l'activité sexuelle des mâles. Il est également possible d'envisager un "effet femelle-femelle» bien que jusqu'à maintenant il n'ait pas été clairement démontré.

Toutefois, le type de structure sociale peut avantager ou non la synchronisation des cycles.
Ainsi, la formation de harems chez les cerfs rouges facilite la synchronisation des oestrus chez les femelles, qu'elle soit induite ou non par un " effet mâle ". Le comportement territorial du chevreuil et la faible sinon nulle tendance grégaire de cette espèce (Sempéré 1978, Sempéré 1979, Sempéré 1982, Sempéré et al 1980) rendent difficile, dans la nature, l'éventualité d'une synchronisation des oestrus. Enfin, l'existence d'un seul moment d'oestrus chez le chevreuil rend problématique les possibilités d'accouplement d'un seul mâle avec un grand nombre de femelles.

\section{b / Induction hormonale de l'oestrus et de l'ovulation}

Les traitements appliqués chez les ovins ont été utilisés chez les cerfs rouges et les daims principalement, quelquefois après de légères adaptations. Ainsi ont été employés les éponges vaginales et les dispositifs intra-vaginaux permettant la libération permanente de progestagène (FGA ou MAP) ou de progestérone. Les durées de traitement sont comprises entre 12 et 15 jours et la quantité de progestérone (ou de progestagène) est parfois légèrement supérieure à celle utilisée chez les ovins.

Pendant la saison de reproduction, l'administration d'un facteur de décharge des hormones hypophysaires endogènes (GnRH) à la fin du traitement progestagène, ou de gonadotropines (PMSG) n'est pas nécessaire pour obtenir oestrus et ovulation (Kelly et al 1982). En revanche, elle est indispensable avant le début de la saison de reproduction (Fisher et al 1986, Fennessy et Fisher 1988). De tels traitements sont utilisables aussi bien chez les cerfs rouges (Adam et al 1985, Moore et Cowie 1986) que chez les daims (Asher et Macmillan 1986, Asher et Smith 1987) et pourraient être généralisés aux autres espèces, y compris le chevreuil. Les quantités de PMSG doivent être définies avec précaution pour éviter des superovulations excessives (Thompson et Asher 1988). Une quantité de PMSG de 500 UI semble être une dose adéquate. Comme chez les ovins et les caprins, la réponse dépend de l'intensité de l'anoestrus; cette réponse est faible chez les femelles allaitantes ou à une période trop éloignée de la saison de reproduction. L'induction d'une activité ovulatoire cyclique est difficile. Quant à la fertilité, elle reste faible. Les variations saisonnières de la production spermatique pourraient être à l'origine en partie de cette faible fertilité.

Chez les femelles ayant des corps jaunes, les prostaglandines seraient capables d'entraîner la régression lutéale quelques jours après l'ovulation (cerf à queue blanche : Haigh 1984 ; chevreuil : Sempéré, résultat non publié).

\section{2 / Traitements photopériodiques : utilisation de la mélatonine}

En fait, les connaissances acquises chez les petits ruminants ont été directement appliquées aux cervidés.

Ainsi, chez les cerfs rouges, l'administration de mélatonine pendant les jours longs de l'été, 
par injection (Webster et Barrell 1985), ingestion (Adam et Atkinson 1984, Nowak et al 1985, Adam et al 1986) ou implant (Fennessy et Fisher 1988) à des femelles, permet d'avancer la date du premier oestrus de la saison de reproduction de 15 à 40 jours. L'intervalle début du traitement - apparition de l'oestrus ou de la première ovulation dépend de différents facteurs: date du début de traitement, état physiologique, présence de mâles traités ou non. Comme chez les ovins, des traitements trop précoces ou trop tardifs seraient sans effet. Le traitement pendant les jours les plus longs (15 juin-15 juillet dans l'hémisphère nord) semble donner des résultats satisfaisants. Les femelles allaitantes ou prépubères répondent moins facilement que les femelles sèches. Enfin, le traitement des mâles en même temps que les femelles est indispensable pour induire un effet mâle et assurer une fertilité normale. Ainsi le traitement des mâles seuls, introduits dans le troupeau de femelles, aboutit à une induction des oestrus aussi précoce que le traitement des seules femelles (Fennessy et Fisher 1988). Le traitement simultané des mâles et femelles est plus efficace.

Les mêmes principes des traitements sont applicables aux espèces se reproduisant en automne ou en hiver. Des résultats similaires à ceux observés chez les cerfs rouges ont été obtenus chez les daims (Asher et al 1987, Asher et al 1988b).

Ces traitements « mélatonine » doivent toutefois être utilisés avec précaution. Le traitement de femelles gravides peut perturber l'initiation de la lactation. Il en résulte une mortalité des jeunes (Asher et al 1988b) probablement liée à la suppression partielle de la sécrétion pré-partum de la prolactine comme cela a été démontré chez les ovins maintenus en jours courts (Ortavant et al 1988). La constitution des réserves corporelles pourrait également être modifiée. Les conséquences à moyen terme (6 mois) doivent être précisées, étudiées avant que ne se généralisent de tels traitements.

Le traitement des seuls mâles peut être une solution intéressante qui mérite plus d'attention puisqu'il est presque aussi efficace que celui des seules femelles.

Enfin, l'induction d'une période de reproduction à contre-saison est probablement possible sur un plan théorique. Elle implique certainement, comme chez les petits ruminants (Chemineau et al 1986), l'alternance de jours longs (lumière) et de "jours courts » (mélatonine). Il est difficile d'envisager à l'heure actuelle, sur des troupeaux, de tels traitements, qui nécessitent des mises au point importantes.

En l'état actuel de nos connaissances, il est prématuré de prévoir l'utilisation de traitements «photopériodiques» simples chez les chevreuils. Le rôle éventuel de la photopériode dans le contrôle de la durée de la diapause embryonnaire doit d'abord être élucidé.

\section{3 / Production, conservation et transfert d'embryons}

Ces techniques n'ont pas fait l'objet à l'heure actuelle de recherches approfondies. Elles peu- vent présenter un intérêt pour la mise en place de certains élevages, l'échange de matériel génétique, la sauvegarde de certaines espèces, etc. Elles sont directement inspirées de celles utilisées chez les bovins, les caprins et les ovins: administration de PMSG ( 1 000 UI) 48 heures avant la fin d'un traitement progestérone ou de FSH ( 2 injections quotidiennes pendant 4 jours, doses décroissantes) à la fin d'un traitement progestérone (Thompson et Asher 1988). Comme pour les autres espèces, les réponses ovulatoires sont très variables d'un individu à l'autre.

Il est prématuré de donner des résultats, de nombreuses conditions restant à préciser.

\section{4 / Insémination artificielle}

Il peut s'agir également d'une technique intéressante dans les cas de synchronisation des oestrus, de production d'embryons, de mise en place de croisements d'absorption (wapiti $x$ cerf rouge, par exemple), de maintien d'espèce en voie de disparition, de programmes d'amélioration génétique.

Plusieurs problèmes doivent être surmontés : production et collecte de sperme, conservation, insémination elle-même.

Le sperme peut être obtenu à l'aide d'un vagin artificiel (renne : Dott et Utsi 1973 ; cerf rouge, chevreuil: Krzywinsky 1987) ou par électro-éjaculation (cerf à queue blanche : Haigh 1984 ; wapiti : Haigh et al 1985 ; daim : Asher et al 1987, Mulley et al 1988).

Les volumes obtenus par électro-éjaculation pendant la période normale de reproduction varient de 0,1 à $4,0 \mathrm{ml}$ suivant les espèces et les individus, et la fréquence des éjaculations. Les concentrations sont comprises alors entre 2 et $3,5 \times 10^{9}$ spermatozoïdes $/ \mathrm{ml}$. Les pourcentages de spermatozoïdes mobiles sont voisins de $80 \%$ et ceux de spermatozoïdes normaux proches de $85 \%$. (Haigh et al 1985, Asher et al 1987).

Le sperme frais est utilisable directement dilué ou non (Dott et Utsi 1973, Haigh 1984, Mulley et al 1988) ou après congélation (Krzywinsky 1987, Mulley et al 1988). Les dilueurs utilisés jusqu'à maintenant ont une composition voisine de celles des dilueurs employés chez les autres espèces.

Les inséminations sont cervicales (comme chez les ovins) ou intra-utérines (Mulley et al 1988) à des moments précis après arrêt d'un traitement de synchronisation des chaleurs ou détection des chaleurs. Les résultats sont encore trop limités pour être généralisés. Ils indiquent clairement cependant que ces techniques sont utilisables.

\section{5 / Conduite de la reproduction}

Le poids des femelles est le facteur le plus important pour l'obtention d'une fertilité élevée. L'éleveur doit donc préparer les femelles à la reproduction en les plaçant dans les meilleures prairies pendant les 2 à 3 mois précédant 
la période des accouplements. Une complémentation peut éventuellement être envisagée.

Les femelles peuvent être mises à la reproduction dès leur deuxième année (15 à 16 mois) dès lors que leur poids représente $75 \%$ du poids des femelles adultes du troupeau. Le sevrage des jeunes pour les femelles allaitantes n'est pas toujours nécessaire.

Les mâles sont également aptes à se reproduire dès l'âge de 15 mois. Cependant leur développement corporel, leur comportement sexuel sont souvent insuffisants. Ils ne doivent être mis à la reproduction qu'avec un nombre limité de femelles.

Les mâles ont besoin d'une préparation alimentaire pour la période de reproduction pendant laquelle ils peuvent perdre jusqu'à $15 \%$ de leur poids (Moore et al 1985) surtout lorsque, comme chez les cerfs rouges, ils tentent de maintenir leur harem et ne consacrent que très peu de temps à leur alimentation.

L'ablation des bois est une sage précaution pour éviter le danger des agressions soit envers l'homme soit envers les autres mâles. Cette intervention n'altère en rien les performances de reproduction (Lincoln et al 1970, Moore et al 1985, Blaxter et al 1988). L'examen de l'intégrité du tractus génital peut être réalisé à cette occasion.

Dans les cas où la paternité doit être connue, un seul mâle peut être utilisé pour 30 à 50 femelles. Pour éviter des problèmes d'infertilité, il doit être remplacé par un autre mâle environ un mois après le début de la période de reproduction.

Dans les cas où plusieurs mâles doivent être employés, des groupes de femelles supérieurs à 60 peuvent être constitués. Il doit alors impérativement être tenu compte des problèmes de dominance. Dans la hiérarchie, les reproducteurs ne doivent pas être trop éloignés les uns des autres (Blaxter et al 1988).

Les mâles peuvent être utilisés pour la reproduction dès l'âge de 2 ans.

L'observation des retours en oestrus est une bonne indication de la fertilité des mâles. La fertilité du troupeau peut être appréciée par d'autres méthodes, par exemple en visualisant les foetus par échographie (Mulley et al 1987, Sempéré et al, résultats non publiés). Toutefois la manipulation d'animaux restés sauvages reste toujours difficile.

Les femelles gravides peuvent être rassemblées en troupeau pour faciliter la surveillance des mise bas et les interventions.

La conduite de la reproduction est probablement plus complexe chez des animaux comme le chevreuil chez lesquels les problèmes de territorialité sont plus marqués que chez les cerfs rouges ou les daims. Les publications sont inexistantes sur la manière de conduire la reproduction dans cette espèce.

\section{Conclusion}

Depuis une vingtaine d'années, les connaissances sur la biologie de la reproduction des cervidés se sont considérablement développées et ont trouvé des applications intéressantes chez les cerfs rouges et les daims, espèces utilisées en élevage dans les zones tempérées. De nouvelles techniques sont en cours de développement, technologie du sperme, insémination artificielle, induction de polyovulations, transfert d'embryons,etc, et pourraient contribuer à une meilleure efficacité de la reproduction et de la sélection.

La physiologie de la reproduction chez les chevreuils reste toutefois encore mystérieuse tout au moins chez les femelles. Des recherches sur la diapause embryonnaire et le contrôle de sa durée sont indispensables pour maîtriser l'élevage de cette espèce. Etre capable de déceler la présence de blastocystes, de provoquer de nouvelles ovulations chez les femelles non fécondées sont des objectifs à atteindre le plus rapidement possible.

Reste à savoir comment ces techniques peuvent être applicables dans des espèces qui restent sauvages et dont la manipulation est difficile.

\section{Références bibliographiques}

ADAM C.L., ATKINSON T., 1984. Effect of feeding mela tonin to red deer (Cervus elaphus) on the onset of the breeding season. J. Reprod. Fert., 72, 463-466.

ADAM C.L., MOIR C.E., ATKINSON T., 1985. Plasma concentrations of progesterone in female red deer (Cervus elaphus) during he breeding season, pregnancy and anoestrus. J. Reprod. Fert., 4, 631-636.

ADAM G.L., MOIR C.E., ATKINSON T., 1986. Induction of early breeding in red deer (Cervus elaphus) by melatonin. J. eprod. Fert., 76, 569-573.

AITKEN R.J., 1974. Delayed implantation in roe deer (Capreolus capreolus). J. Reprod. Fert., 39, 225-233.

AITKEN R.J., 1981. Aspects of delayed implantation in the roe deer (Capreolus capreolus). J. Reprod. Fert. Suppl. 29, 83-95.

ARENDT J., 1986. Role of the pineal gland and melatonin in seasonal reproductive functions in mammals. Oxford Rev, Reprod. Biol., 8, 266-320.

ARMAN P., HAMILTON W.J., SHARMAN G.A.M., 1978. Observations on the calving of free-ranging tame reddeer (Cervus elaphus). J. Reprod. Fert., 54, 279-283.

ASHER G.W., 1985. Oestrous cycle and breeding season of farmed fallow deer, Dama dama. J. Reprod. Fert., 75 521-529.

ASHER G.W., ADAM J.L., 1985. Reproduction of farmed red and fallow deer in northern New Zealand. In "Biology of Deer Production ", The Royal Society of New Zealand, Bulletin 22, 217-224

ASHER G.W., MacMILLAN K.R., 1986. Induction of oestrus and ovulation in farmed fallow deer (Dama dama) by using progesterone and GnRH treatment. J. Reprod. Fert., 78, 693-697.

ASHER G.W., SMITH J.F., 1987. Induction of oestrus and ovulation in farmed fallow deer (Dama dama) by using progesterone and PMSG treatment. J. Reprod. Fert., 81 113-118.

ASHER G.W, BARRELL G.K, PETERSON A.J. 1986. Hormonal changes around oestrus of farmed fallow deer Dama dama. J. Reprod. Fert., 78, 487-496.

ASHER G.W., DAY A.M., BARRELL G.K., 1987. Annua cycle of liveweight and reproductive changes of farmed male fallow deer (Dama dama) and the effect of daily ora administration of melatonin in summer on the attainment of seasonal fertility. J. Reprod. Fert., 79, 353-362.

ASHER G.W., DAY A.M., BARRELL G.K., 1987. Annual cycle of liveweight and reproductive changes of farmed male fallow deer (Dama dama) and the effect of daily ora administration of melatonin in summer on the attainment of seasonal fertility. J. Reprod. Fert., 79, 353-362. 
ASHER G.W., PETERSON A.J., WATKINS W.B., 1988 a. Hormonal changes during luteal regression in farmed fallow deer, (Dama dama). J. Reprod. Fert., 84, 379-386. ASHER G.W., BARRELL G.K., ADAM J.L., STAPLES L.D., 1988b. Effects of subcutaneous melatonin implants on reproductive seasonality of farmed fallow deer (Dama dama). J. Reprod. Fert., 84, 679-691.

BARRELL G.K., MUIR P.D., SYKES A.R. 1985. Seasonal profiles of plasma testosterone, prolactin, and growth hormone in red deer stags. In "Biology of Deer Production ". The Royal Society of New Zealand, Bulletin 22, 185-190.

BLAUEL G., 1936. Beobachtungen über die Enstehung der Perücke beim Rehbock. 2. Endokrinologië, 17, 369372.

BLAXTER K., KAY R.N.B., SHARMAN G.A.M., CUNNINGHAM J.M.M., EADIE J., HAMILTON W., 1988. In «Farming the red deer", pp 31-46. HMSO Publications Centre.

BRAMLEY P.S., 1970, Territoriality and reproductive behaviour of roe deer. J. Reprod. Fert., Suppl. 11, 43-70.

BRAY A.R., KELLY R.W., 1979. Mating management and reproductive activity of intensively farmed red deer. Proc. N. Z. Soc. Anim. Prod., 39, 94-99.

BRONSON F.H., 1985. Mammalian reproduction : an ecological perspective. Biol. Reprod., 32, 1-26.

BROWN R.D., COWAN R.I., KAVANAUGH E.J.F., 1978. Effect of pinealectomy on seasonal androgen titres, antler growth and feed intake in white-tailed deer. J. Anim. Sci., $47,435-440$

BROWN W.B., FORBES J.M., GOODALL E.D., KAY R.N.B., SIMPSON A.M., 1979. Effects of photoperiod on food intake, sexual condition and hormone concentrations in stags and rams. J. Physiol., 296, 58-59P (Abstr.) BROWN R.D., CHAO C.C., FAULKNER L.W., 1983. The endocrine control of the initiation and growth of antlers in white-tailed deer. Acta Endocrinologica, 103, 138-144. BUBENIK G.A., 1983. Shift of seasonal cycle in whitetailed deer by oral administration of melatonin. J. Exp. Zool., 225, 155-156.

BUBENIK G.A.. 1986. Regulation of seasonal endocrine rhythms in male boreal cervids. In " Endocrine regulations as adaptative mechanisms to the environment ", Assenmacher I. et Boissin J. Eds, C.N.R.S., Paris, pp 461474

BUBENIK G.A., SMTTH P., 1985. Effect of orally administered melatonin on circannual rhythms of male deer. In "Biology of Deer Production ", The Royal Society of New Zealand, Bulletin 22, 191-192.

BUBENIK G.A., MORRIS J.M., SCHAMS D., CLAUS A., 1982. Photoperiodicity and circannual levels of LH, FSH, and testosterone in normal and castrated male, whitetailed deer. Can. J. Physiol. Pharmacol., 60, 788-793.

BUBENIK G.A., SCHAMS D., LEATHERLAND J.F., 1985. Seasonal rhythms of prolactin and its role in the antler cycle of white-tailed deer. In "Biology of Deer Production ", The Royal Society of New Zealand, Bulletin 22, $257-262$

BUDDE W.S., 1983. Effects of photoperiod on puberty attainment of female white-tailed deer. J. Wildl. Manage. $47,595-604$

CHAPLIN R.E., WHITE R.W., 1972. The influence of age and season on the activity of the testes and epididymides of the fallow deer, (Dama dama). J. Reprod. Fert., 30, 361369.

CHAPMAN D.I., 1974. Reproductive physiology in relation to deer management. Mammal Review, 4, 61-74.

CHARDONNET P., 1983. Exploitation rationelle des cervidés en Nouvelle-Zélande. Situation mondiale, gestion, pathologie. Thèse Méd. vét. Alfort, $n^{\circ} 149,187 \mathrm{p}$.

CHEMINEAU P., NORMANT E., RAVAULT I.P., THIMONIER J., 1986. Induction and persistence of pituitary and ovarian activity in the out-of-season lactating dairy goat after a treatment combining a skeleton photoperiod, melatonin and the male effect. J. Reprod. Fert., 78, 497504.

CLUTTON-BROCK T., GUINNESS F.E., 1975. Behaviour of red deer (Cervus elaphus L.) at calving time. Behaviour, 55, 287-300.

CLUTTON-BROCK T., ALBON S.D., GUINNESS F., 1981. Parental investment in male and female offspring in polygenous mammals. Nature, 289, 487-489.
COLLIN J.P., ARENDT J., GERN W.A., 1988. Le « troisième œil ». La Recherche, 19, 1154-1165.

CURLEWIS J.D., LOUDON A.S.I., COLEMAN A.P.M., 1988. Oestrus cycles and the breeding season of the Père David's beer hind (Elaphurus davidianus). J. Reprod. Fert., 82, 119-126.

DE VOS A., 1982. Deer farming. Guidelines on practical aspects. F.A.O. Animal Production and Health Paper, $n^{\circ}$ 27, 54 p., The F.A.O. Technical Papers, Rome.

DOTT H.M., UTSI M.N.P., 1973. Artificial insemination of reindeer (Rangifer tarandus]. J. Zool., Lond., 170, 505508.

FENNESSY P.F., FISHER M.W, 1988. Advancing the calving season in red deer. Proc. Deer Course for Veterinarians, 5 , in press.

FENNESSY P.F, SUTTIE I.M., CROSBIE S.F., CORSON I.D., ELGAR H.J., LAPWOOD K.R., 1988. Plasma LH and testosterone responses to gonadotrophin-releasing hormone in adult red deer (Cervus elaphus) stags during the annual antler cycle. J. Endocr., 117, 35-41.

FISHER M.W., FENNESY P.F., SUTTIE J.M., CORSON I.D., PEARSE A.J.T., DAVIS G.H., JOHNSTONE P.D. 1986. Early induction of ovulation in yearling red deer hinds. Proc. N. Z. Soc. Anim. Prod., 46, 171-173.

FLETCHER J., 1974. The timing of reproduction in red deer, (Cervus elaphus) in relation to latitude. J. Zool. Lond., 172, 363-367.

GOSS R.J., 1969. Photoperiodic control of antler cycles in deer. I. Phase shift and frequency changes. J. Exp. Zool, $170,311-324$

GOSS R.J., 1985. Tissue differentiation in regenerating antlers.In "Biology of Deer Production », The Royal Society of New Zealand, Bulletin 22, 229-238.

GOSS R.J., ROSEN J.K., 1973. The effect of latitude and photoperiod on the growth of antlers. J. Reprod. Fert. Suppl. 19, 111-118.

GUINNESS F., LINCOLN G.A., SHORT R.V., 1971. The reproductive cycle of the female red deer, (Cervus elaphus L.). J. Reprod. Fert., 27, 427-438.

GUINNESS F.E., ALBON S.D., CLUTTON-BROCK T.H., 1978. Factors affecting reproduction in red deer (Cervus elaphus) hinds on Rhum. J. Reprod. Fert., 54, 325-334.

HAIGH J.C., 1984. Artificial insemination of two whitetailed deer. J. Am. Vet. Med. Assoc., 185, 1446

HAIGH J.C., CATES W.F., GLOVER G.I., RAWLINGS N.C., 1984. Relationships between seasonal changes in serum testosterone concentrations, scrotal circumference and sperm morphology of male wapiti (Cervus elaphus). J. Reprod. Fert., 70, 413-418.

HAIGH J.C., BARTH A.D., CATES W.F., GLOVER G.J., 1985. Electro-ejaculation and semen evaluation of wapiti. In "Biology of Deer Production », The Roval Society of New Zealand, Bulletin 22, 197-203.

HAMILTON W.J., BLAXTER K.L., 1980. Reproduction in farmed red deer. I. Hind and stag fertility. J. Agric. Sci., Camb., 95, 261-273.

HARDER J.D., WOOLF A., 1976. Changes in plasma levels of oestrone and oestradiol during pregnancy and parturition in white-tailed deer. J. Reprod. Fert., 47, 161163.

HOCHEREAU de REVIERS M.T., LINCOLN G.A., 1978. Seasonal variations in the histology of the testis of the red deer, (Cervus elaphus). J. Reprod. Fert., 54, 209-213.

HOFFMANN B., BARTH D., KARG H., 1978. Progesterone and estrogen levels in peripheral plasma of the pregnant and nonpregnant roe deer (Capreolus capreolus). Biol, reprod., 19, 931-935.

KARSCH F.., BITTMANN E.L., FOSTER D.L., GOODMAN R. LEGAN S.J., ROBINSON J E, 1984 Neuroendocrine basis of seasonal reproduction. Recent Prog. Horm. Res., 40, 185-232.

KAY R.N.B., RYDER M.L., 1978. Coat growth in red deer (Cervus elaphus) exposed to a day-length of six months duration. J. Zool., Lond., 185, 505-510.

KELLY R.W. CHALLIES C.N., 1978. Incidence of ovulation before the onset of the rut and during pregnancy in red deer hinds. N. Z. J. Zool., 5, 817-819.

KELLY R.W., MOORE G.H., 1977. Reproductive performance in farmed red deer. N. Z. Agric. Sci., 11, 179-181.

KELLY R.W., WHATELEY J.A., 1975. Observations on the calving of red deer (Cervus elaphus) run in confined areas. Applied Animal Ethology, 1, 293-300. 
KELLY R.W., McNATTY K.P., MOORE G.H., ROSS D. GIBB M., 1982. Plasma concentrations of LH, prolactin, oestradiol and progesterone in female red deer (Cervus elaphus) during pregnancy. J. Reprod. Fert., 64, 475-483.

KELLY R.W., McNATTY K.P., MOORE G.H., 1985. Hormonal changes about oestrus in female red deer. In "Biology of Deer Production », The Royal Society of New Zealand, Bulletin 22, 181-184.

KIRKWOOD J.K., GASKIN C.D., MARKHAM J., 1987 Perinatal mortality and season of birth in captive wild ungulates. Vet. Rec., 120, 386-390.

KRZYWINSKY A., 1987. Artificial insemination and embryo transfer in deer : applying these methods for propagating Endangered species. In "Biology and management of the Cervidae », C.M. Wemmer Ed., Smithsonian Institution Press, Whasinghton, D.C., pp 443-449.

LEADER-WILLIAMS N., 1979. Age related changes in the testicular and antler cycles of reindeer, Rangifer tarandus. J. Reprod. Fert., 57, 117-126.

LEADER-WILLIAMS N., ROSSER A.M., 1983. Ovarian characteristics and reproductive performance of reindeer, Rangifer tarandus. J. Reprod. Fert., 67, 247-256.

LINCOLN G.A., 1971. The seasonal reproductive changes in the red deer stag (Cervus elaphus). J. Zool., Lond., 163, $105-123$

LINCOLN G.A., 1985. Seasonal breeding in deer. In "Biology of Deer Production ", The Royal Society of New Zealand, Bulletin 22, 165-179.

LINCOLN G.A., GUINNESS F.E., 1972. Effect of altered photoperiod on delayed implantation and moulting in roe deer. J. Reprod. Fert., 31, 455-457.

LINCOLN G.A., GUINNESS F.E., 1973. The sexual significance of the rut in red deer. J. Reprod. Fert., Suppl. 19, $475-489$

LINCOLN G.A., KAY R.N.B., 1979. Effects of season on the secretion of LH and testosterone in intact and castrated red deer stags (Cervus elaphus). J. Reprod. Fert., $55,75-80$

LINCOLN G.A., SHORT R.V., 1980. Seasonal breeding : nature's contraceptive. Recent Prog. Horm. Res., 36, 1-52.

LINCOLN G.A., YOUNGSON R.W. SHORT RV, 1970 The social and sexual behaviour of the red deer siag. J. Reprod. Fert., Suppl. 11, 71-103.

LINCOLN G.A., FRASER H.M., FLETCHER T.I., 1984 Induction of early rutting in male red deer (Cervus elaphus) by melatonin and its dependence on LHRH. J. Reprod. Fert., 72, 339-343.

LOUDON A.S.I., CURLEWIS J.D;; 1988. Cycles of antler and testicular growth in an aseasonal tropical deer (Axis axis). J. Reprod. Fert., 83, 729-738.

LOUDON A.S.I., MCNEILLY A.S., MILNE J.A., 1983. Nutrition and lactational control of fertility in red deer Nature, London, 302, 145-147.

MacKENZIE A.R., 1985. Reproduction of farmed Rusa deer (Cervus timorensis) in south-east Queensland, Australia. In "Biology of Deer Production», The Royal Society of New Zealand, Bulletin 22, 213-215.

McCOMB K., 1987. Roaring by red deer stags advances the date of oestrus in hinds. Nature, London, 330, 648 649.

MCMILLIN JM SEAL US, KEENLYNE K.D., ERICKSON A.W., JONES J.E., 1974. Annual testosterone rhythm in the adult white- tailed (Odocoileus virginianus borealis). Endocrinology, 94, 1034-1040.

MIRARCHI R.E., SCANLON P.F., KIRKPATRICK R.L. 1977. Annual changes in spermatozoan production and associated organs of white-tailed deer. J. Wildl. Manage. 41, 92-99.

MITCHELL B,, 1973. The reproductive performance of wild scottish red deer, Cervus elaphus. J. Reprod. Fert., Suppl. 19, 271-285.

MITCHELL B., BROWN D., 1974. The effects of age and body size on fertility in female red deer (Cervus elaphus L.). XIth Int. Congr. Game Biol., 13E, 89-98.

MOORE G.H., COWIE G.M., 1986. Advancement of breeding in non-lactating adult red deer hinds. Proc. N. Z. Soc. Anim. Prod., 46, 175-178.

MOORE G.H., COWIE G.M., BRAY A.R., 1985. Herd management of farmed red deer. In « Biology of Deer Production », The Royal Society of New Zealand, Bulletin 22 343-355
MORGAN P.D., ARNOLD G.W., LINDSAY D.R., 1972. A note on the mating behaviour of ewes with various senses impaired. J. Reprod. Fert., 30, 151-152.

MULLEY R.C., ENGLISH A.W., RAWLINSON R.J. CHAPPLE R.S., 1987. Pregnancy diagnosis of fallow deer by ultrasonog raphy. Aust. Vet. J., 64, 257-258.

MULLEY R.C., MOORE N.W., ENGLISH A.W., 1988. Successful uterine insemination of fallow deer with fresh and frozen semen. Theriogenology, 29, 1149-1153.

NOWAK R., ELMHIRST R.N., RODWAY R.G., 1985. A note on the effect of melatonin feeding on the initiation of ovarian activity and on plasma prolactin levels in lactating and non- lactating red deer hinds. Anim. Prod., 40, 515-518.

ORTAVANT R., BOCQUIER F., PELLETIER I., RAVAULT J.P., THIMONIER I., VOLLAND-NAIL P., 1988. Seasonality of reproduction in sheep and its control by photoperiod. (In « Controlled breeding of sheep : a tribute to T.J. Robinson ", J.K. Findlay Ed., C.S.I.R.O., Australie), Aust. J. Biol. Sci., 41, 69-85.

PELLETIER J., 1986. Contribution of increasing and decreasing daylength to the photoperiodic control of $\mathrm{LH}$ secretion in the Ile-de-France ram. J. Reprod. Fert., 77, $505-512$

PLOTK, F.D., SEAL U.S., SCHMOLLER G.C., KARNS P.D., KEENLYNE K.D., 1977a. Reproductive steroids in the white-tailed deer (Odocoileus virginianus borealis). I. Seasonal changes in the female. Biol. Reprod., 16, 340343.

l'i(jh.1 H.D., SEAL U.S., VERME L.J., OZOGA J.J., 1977b. Reproductive steroids in the white-tailed deer (Odocoileus virginianus borealis). II. Progesterone and estrogen levels in peripheral plasma during pregnancy. Biol. Reprod., 17, 78-83.

PLOTKA E.D., SEAL U.S., VERME L.J., OZOGA J.J., 1980. Reproductive steroids in deer. III. Luteinizing hormone, estradiol and progesterone around estrus. Biol. Reprod., 22, 576-581.

PLOTKA E.D., SEAL U.S., VERME L.J., OZOGA J.J., 1982. Reproductive steroids in white-tailed deer. III. Origin of progesterone during pregnancy. Biol. Reprod. $26,258-262$.

POLLOCK A.M., 1975. Seasonal changes in appetite and sexual condition in red deer stags maintained on a six month photoperiod. J. Physiol., 244, 95-96P.

RAVAULT J.P., THIMONIER J., 1988. Melatonin patterns in ewes maintained under skeleton or resonance photoperiodic regimens. Reprod. Nutr. Dévelop., 28, 473-486.

SADLEIR R.M.F.S., 1987. Reproduction of female Cervids. In "Biology and management of the Cervidae", C.M. Wemmer Ed., Smithsonian Institution Press, Whasinghton, D.C., pp 123-144.

SCHAMS D., BARTH D., 1982. Annual profiles of reproductive hormones in peripheral plasma of the male roe deer (Capreolus capreolus). J. Reprod. Fert., 66, 463-468. SCHAMS D., BARTH D., KARG H., 1980. LH, FSH and progesterone concentrations in peripheral plasma of the female roe deer (Capreolus capreolus) during the rutting season. J. Reprod. Fert., 60, 109-114.

SCHULTE B.A., SEAL U.S., PLOTKA E.D., LETELLIER M.A., VERME L.J., OZOGA J.J., PARSONS J.A., 1981. The effect of pinealectomy on seasonal changes in prolactin secretion in the white-tailed deer (Odocoileus virginianus). Endocrinology, 108, 173-178.

SEMPERE A., 1977. Plasma progesterone levels in the roe deer (Capreolus capreolus L.). ]. Reprod. Fert., 30, 365366.

SEMPERE A, 1978. The annual cycle of plasma testosterone and territorial behavior in the roe deer. Environmental Endocrinology. I. Assenmacher et D.S. Farner Eds, Springer- Verlag, Berlin, pp 73-74.

SEMPERE A., 1979. Utilisation et évolution du domaine vital chez le chevreuil mâle européen déterminé par radiotracking, Biol. Behav., 1, 75-87.

SEMPERE A., 1982. Fonction de reproduction et caractères sexuels secondaires chez le chevreuil (Capreolus capreolus L.) : Variations saisonnières et incidences sur l'utilisation du budget temps-espace. Thèse Doct. ès Sci., Université de Tours, $291 \mathrm{pp}$

SEMPERE A., 1988. The annual antler cycle of the european roe deer (Capreolus capreolus) in relationship to the reproductive cycle. In "Horns and antlers", G.A. Bubenik et A.B. Bubenik Eds, Junk Publishing Company, Pays-Bas. (sous presse). 
SEMPERE A.J.. BOISSIN J.. 1982. Neuroendocrine and endocrine control of testicular activity and the antler cycle from birth to adulthood in the male roe deer (Capreolus capreolus L.). Int. Symp. on Antler Development in Cervidae. Kingsville, Texas. pp 109-122.

SEMPERE A.J., LACROIX $\Lambda .$, 1982. Temporal and seasonal relationships between LH, testosterone and antlers in fawn and adult male roe deer (Capreolus capreolus L.) : a longitudinal study from birth to four years of age. Acta Endocrinologica, 99, 295-301.

SEMPERE A., GARREAU J.J., BOISSIN J., 1980. Variations saisonnières de l'activité de marquage territorial el de la testostéronémie chez le Chevreuil mâle adulte (Capreolus capreolus L.). C. R. Acad. Sci., 290, série D, 803-806.

SEMPERE A.J., BOISSIN I., DUTOURNE, B., LACROIX A., BLANC M.R, RAVAULT J.P., 1983. Variations de la concentration plasmatique en prolactine. LH et FSH el de l'activité testiculaire au cours de la première année de vie chez le chevreuil (Capreolus capreolus l.). Gen. Comp. Endocrinol., 52, 247-254.

SEMPERE A. NOEL A.M. BOUTIN J.M., 1988. Etude comparative de l'influence des variations saisonnières de la progestéronémie et de la lactation sur le développement des tétines chez le chevreuil (Capreolus capreolus L.) : aspects fondamental et appliqué. Gibier Faune Sauvage, 5 , sous presse.

SHORT R.V., HAY M.F., 1966. Delayed implantation in the roe deer (Capreolus capreolus). Symp. zool. Soc: Lond., 15, 173-194.

SHORT R.V., MANN T., 1966. The sexual cycle of a seasonally breeding mammal, the roebuck (Capreolus capreolus). J. Reprod. Fert., 12, 337-351.

SIMPSON A.M. SUTTIE J.M. KAY R N.B, 1983/1984 The influence of artificial photoperiod on the growth. appetite and reproductive status of male red deer and sheep. Anim. Reprod. Sci., 6, 291-299.

SNYDER D.L., COWAN R.L., HAGEN D.R., SCHANBACHER B.D., 1983. Effect of pinealectomy on seasonal changes in antler growth and concentrations of testosterone and prolactin in white-tailed deer. Biol. Reprod., 29, 63-71.

SUTTIE J.M., LINCOLN G.A., KAY R.N.B., 1984a. Endocrine control of antler growth in red deer stags. J. Reprod. Fert., 71, 7-15.
SUTTIF I.M.. CORSON I.D., FENNESSY P.F., 1984b. Voluntary intake, testis development and antler growth pat terns of male deer under a manipulated photoperiod. Proc. N. Z. Soc. Anim. Prod., 44, 167-170

THERIEZ, M.. 1988. L'élevage et l'alimentation du cerf (Cervus elaphus). I. Caractéristiques physiologiques, besoins alimentaires el élevage des adultes. INRA Prod. Anim, 1 (5), 319-330.

THIMUNITR J., TERQUI M., CHEMINEAU P., 1986. Conduite de la reproduction des petits ruminants dans les différenles parties du monde. In " Nuclear and related Techniques in animal Production and I lealth ». Proceedings of an International Svmposium. 1.A.E.A. - F.A.O.. Vienne, Mars 17-21, I.A.E.A.-S.M. 292/17, 135-147.

THOMAS D.C., COWAN I. McT., 1975. The pattern of reproduction in female Columbian black-tailed deer. Odocoileus hemionus columbianus. I. Reprod. Fert., 44 $261-272$.

THOMPSON J.G.E., ASHER G.W., 1988. Superovulation and ova recovery in farmed fallow deer (Dama dama Proc. Aust. Soc. Reprod. Biol., 20, 4 (Abstr.)

WEBB J.W., NELLIS D.W., 1981. Reproductive cycle of white taileddeer of SI Croix, Virgin Islands. J. Wildl. Manage., 45, 253-258.

WEBSTER J.R., BARRELLL G.K. 1985. Advancement of reproductive activity, seasonal reduction in prolactin secretion and seasonal pelage changes in pubertal red deer hinds (Cervus elaphus) subjected to artificially shortened daily photoperiod or daily melatonin treatments. J. Reprod. Fert., 73, 255-260.

WEMMER C.W., 1983. Sociology and management. In "The biology and management of an extinct species, Père David's deer. B.B. Beck and C.W. Wemmer Fds Noyes Publications, New Jersey.

WESSON J.A., III, SCANLON P.F, KIRKPATRICK R.L. MOSBY II.S., BUTCIIER R.L., 1979. Influence of chemical immobilization and physical restraint on steroid hormone levels in blood of white-tailed deer. Can. I. Zool., $57,768-776$

WHITFHEAD G.K., 1972. Appendix : Classification of the Corvidae. In: Deer of the world, 151-169. Constable et Company Ltd, London. 